\title{
ALGORITHMS FOR MULTIDIMENSIONAL SPECTRAL FACTORIZATION AND SUM OF SQUARES
}

\author{
D. Napp Avelli and H. L. Trentelman *
}

June 3, 2007

\begin{abstract}
In this paper, algorithms are developed for the problems of spectral factorization and sum of squares of polynomial matrices with $n$ indeterminates, and a natural interpretation of the tools employed in the algorithms is given using ideas from the theory of lossless and dissipative systems. These algorithms are based on the calculus of $2 n$-variable polynomial matrices and their associated quadratic differential forms, and share the common feature that the problems are lifted from the original $n$-variable polynomial context to a $2 n$-variable polynomial context. This allows to reduce the spectral factorization problem and the sum of squares problem to linear matrix inequalities (LMI's), to the feasibility of a semialgebraic set or to a linear eigenvalue problem.
\end{abstract}

keywords: polynomial multidimensional spectral factorization, two-variable polynomial matrices, quadratic differential forms, dissipativity, sum of squares, linear matrix inequalities.

\section{Introduction}

The spectral factorization problem was originally used by Wiener in [17] to obtain a frequency domain solution to optimal filtering problems. Later it has arisen in different areas of systems and control, such as the polynomial and behavioral approaches to $H_{\infty}$ control problems, theory of dissipative systems, and in LQG theory (see for instance [4]). The related problem of sum of squares (SOS) has appeared earlier and has a venerable history. It has connections with Hilbert's 17th problem, that he posed at the International Congress of Mathematicians in 1900. Recently, much attention has been directed to SOS methods in order to test feasibility of semialgebraic sets [8], and to the problem of finding Lyapunov functions for nonlinear systems [10].

Most of the vast literature on these problems deal with either the 1-dimensional case (i.e., the case of polynomials in one indeterminate) or the scalar case (as opposed to the matrix case). In this paper we present a unified framework to address both the problem of spectral factorization and the problem of SOS for polynomial matrices, giving gentle and systematic algorithms. Also, a natural interpretation of the tools employed in the algorithms is given using ideas from the theory of lossless and dissipative systems. In this

\footnotetext{
${ }^{*}$ Research Institute for Mathematics and Computing Science, P.O. Box 800, 9700 AV Groningen, The Netherlands, tel: 31-50-3633998, fax: 31-50-3633976 , e-mail: h.l.trentelman@math.rug.nl, diego@math.rug.nl
} 
paper we will pay particular attention to the theory of dissipative distributed systems, as recently developed in [12]. This theory is underlying most of our algorithms and proofs, where the problem is lifted from the original $n$-variable polynomial context to a $2 n$-variable polynomial context. The key is that factorizations of $2 n$-variable polynomial matrices can essentially be performed by doing factorizations of constant real symmetric matrices. Similar ideas were used in [15] for spectral factorization of polynomial matrices in one variable and in [13] for SOS of polynomials in $n$ variables.

The problem of multidimensional spectral factorization is stated as follows. Denote the $n$-dimensional indeterminate by $\xi=\left(\xi_{1}, \xi_{2}, \ldots, \xi_{n}\right)$. Given a para-hermitian real $q \times q$ $n$-variable polynomial matrix $Z(\xi)$, i.e. a real $n$-variable polynomial matrix with the property that $Z^{T}(-\xi)=Z(\xi)$, the problem is to compute another real $n$-variable polynomial matrix $F(\xi)$ (called a spectral factor) such that $Z(\xi)=F^{T}(-\xi) F(\xi)$. We also address the case when $F(\xi)$ is allowed to be a rational polynomial matrix.

The problem of sum of squares is stated here as follows. Given a real symmetric positive semidefinite $q \times q n$-variable polynomial matrix $Z(\xi)$, i.e. a real $n$-variable polynomial matrix with the property that $Z(\xi)^{T}=Z(\xi)$ and $Z(\xi) \geq 0$ for all $\xi \in \mathbb{R}^{n}$, the problem is to compute another $n$-variable polynomial matrix $F(\xi)$ such that $Z(\xi)=F^{T}(\xi) F(\xi)$.

This paper is organized as follows. Section 2 contains the basic background material on multidimensional systems, quadratic differential forms and $2 n$-variable polynomial matrices. Sections 3 and 4 connect the problem of polynomial spectral factorization with $2 n$-variable polynomial matrices and the problem of factorizing a constant matrix that is obtained from solving a linear matrix inequality (LMI). Also, in section 4, we reduce the problem of rational spectral factorization to the problem of finding a feasible point of a semialgebraic set and to a linear eigenvalue problem. Finally, in section 5 , we connect the sum of squares problem to $2 n$-variable polynomial matrices and linear matrix inequalities.

\section{$2 n \mathbf{D}$ multidimensional systems and $2 n$-variable polynomial matrices}

In the behavioral approach to system theory, the behavior is a subset of the space $\mathbb{W}^{\mathbb{T}}$ consisting of all trajectories from $\mathbb{T}$, the indexing set, to $\mathbb{W}$, the signal space. In this paper we consider systems with $\mathbb{T}=\mathbb{R}^{n}$ (from which the terminology 'nD-system' derives) and $\mathbb{W}=\mathbb{R}^{q}$. We call $\mathfrak{B}$ a linear differential $n D$ behavior if it is the solution set of a system of linear, constant-coefficient partial differential equations, more precisely, if $\mathfrak{B}$ is the subset of $\mathfrak{C}^{\infty}\left(\mathbb{R}^{n}, \mathbb{R}^{q}\right)$ (the set of smooth functions) consisting of all solutions to

$$
R\left(\frac{d}{d x}\right) w=0
$$

where $R$ is a polynomial matrix in the $n$-dimensional indeterminate $\xi=\left(\xi_{1}, \xi_{2}, \ldots, \xi_{n}\right)$, and $\frac{d}{d x}=\left(\frac{\partial}{\partial x_{1}}, \ldots, \frac{\partial}{\partial x_{n}}\right)$. We call $(1)$ a kernel representation of $\mathfrak{B}$ and write $\mathfrak{B}=\operatorname{ker}(\mathrm{R})$. In general, there are many other ways to represent an $n \mathrm{D}$ system. Let $\mathfrak{B}$ be the subset of $\mathfrak{C}^{\infty}\left(\mathbb{R}^{n}, \mathbb{R}^{q}\right)$ consisting of all functions $w$ for which there exits $\ell \in \mathfrak{C}^{\infty}\left(\mathbb{R}^{n}, \mathbb{R}^{q}\right)$ such that

$$
w=M\left(\frac{d}{d x}\right) \ell .
$$

Here $M$ is a polynomial matrix in the indeterminate $\xi=\left(\xi_{1}, \xi_{2}, \ldots, \xi_{n}\right)$, and again $\frac{d}{d x}=$ $\left(\frac{\partial}{\partial x_{1}}, \ldots, \frac{\partial}{\partial x_{n}}\right)$. We call $(2)$ an image representation of $\mathfrak{B}$ and the variable $\ell$ is called the 
latent variable. In this case we write $\mathfrak{B}=\operatorname{im}(M)$. Image representations often appear in physics, where the latent variables in a such a representation are called potentials. An important propery of behaviors is controllability, see [11]. It can be shown that a behavior admits an image representation if and only if it is controllable.

In many modelling and control problems for linear systems it is necessary to study quadratic functionals of the system variables and their derivatives, for example, in linear quadratic optimal control and $H_{\infty}$-control. We will use polynomial matrices in $2 n$ variables as a tool to express quadratic functionals of functions of $n$ variables (see [18] and [12]). We will now review the notational conventions for quadratic differential forms and discuss the results that are relevant to the problems of multivariable spectral factorization and sums of squares.

For convenience, denote $x:=\left(x_{1}, \ldots, x_{n}\right)$. Multi-indices are denoted by $k:=\left(k_{1}, \ldots, k_{n}\right)$ and $l:=\left(l_{1}, \ldots, l_{n}\right)$, and indeterminates by $\zeta:=\left(\zeta_{1}, \ldots, \zeta_{n}\right)$ and $\eta:=\left(\eta_{1}, \ldots, \eta_{n}\right)$. We also denote $\zeta^{k}:=\zeta_{1}^{k_{1}} \zeta_{2}^{k_{2}} \cdots \zeta_{n}^{k_{n}}$ and $\eta^{k}:=\eta_{1}^{k_{1}} \eta_{2}^{k_{2}} \cdots \eta_{n}^{k_{n}}$. Let $\mathbb{R}^{q \times q}[\zeta, \eta]$ denote the set of real polynomial $q \times q$ matrices in the $2 n$ indeterminates $\zeta$ and $\eta$; that is, an element of $\mathbb{R}^{q \times q}[\zeta, \eta]$ is of the form

$$
\Phi(\zeta, \eta)=\sum_{k, l} \Phi_{k, l} \zeta^{k} \eta^{l}
$$

where $\Phi_{k, l} \in \mathbb{R}^{q \times q}$; the sum ranges over the multi-indices $k, l \in \mathbb{N}^{n}$, and is assumed to be finite. The $2 n$-variable polynomial matrix $\Phi(\zeta, \eta)$ is called symmetric if $\Phi(\zeta, \eta)=\Phi(\eta, \zeta)^{T}$, equivalently if $\Phi_{k, l}=\Phi_{l, k}^{T}$ for all $l$ and $k$. In this paper we restrict our attention to the symmetric elements in $\mathbb{R}^{q \times q}[\zeta, \eta]$, and denote this subset by $\mathbb{R}_{s}^{q \times q}[\zeta, \eta]$. Any symmetric $\Phi$ induces a quadratic functional

$$
\begin{aligned}
& Q_{\Phi}: \mathfrak{C}^{\infty}\left(\mathbb{R}^{n}, \mathbb{R}^{q}\right) \longrightarrow \mathfrak{C}^{\infty}\left(\mathbb{R}^{n}, \mathbb{R}\right) \\
& Q_{\Phi}(w):=\sum_{k, l}\left(\frac{d^{k} w}{d x^{k}}\right)^{T} \Phi_{k, l} \frac{d^{l} w}{d x^{l}}
\end{aligned}
$$

where the $k$-th derivative operator $\frac{d^{k}}{d x^{k}}$ is defined as $\frac{d^{k}}{d x^{k}}:=\frac{\partial^{k_{1}}}{\partial x_{1}^{k_{1}}} \ldots \frac{\partial^{k_{n}}}{\partial x_{n}^{k_{n}}}$ (similarly for $\frac{d^{l}}{d x^{l}}$ ). We will call $Q_{\Phi}$ the quadratic differential form (in the following abbreviated with QDF) associated with $\Phi$. For a given symmetric $2 n$-variable polynomial matrix $\Phi(\zeta, \eta)=$ $\sum_{k, l} \Phi_{k, l} \zeta^{k} \eta^{l}$, the $n$-tuple $k \in \mathbb{N}^{n}$ is called the degree of the monomial $\zeta^{k}:=\zeta_{1}^{k_{1}} \zeta_{2}^{k_{2}} \cdots \zeta_{n}^{k_{n}}$. In the 1 -variable case $(n=1)$ it is of course clear how to order the monomials $\zeta^{k}$ with increasing degree. In that case one can write

$$
\Phi(\zeta, \eta)=\left(I_{q}, \zeta I_{q}, \ldots, \zeta^{N} I_{q}\right)\left(\begin{array}{cccc}
\Phi_{0,0} & \Phi_{0,1} & \ldots & \Phi_{0, N} \\
\Phi_{1,0} & \Phi_{1,1} & \ldots & \Phi_{1, N} \\
\vdots & \vdots & \ddots & \vdots \\
\Phi_{N, 0} & \Phi_{N, 1} & \ldots & \Phi_{N, N}
\end{array}\right)\left(\begin{array}{c}
I_{q} \\
\eta I_{q} \\
\vdots \\
\eta^{N} I_{q}
\end{array}\right)
$$

where the symmetric matrix in the middle is called the coefficient matrix of $\Phi(\zeta, \eta)$, denoted by $\operatorname{mat}(\Phi)$. In order to define the coefficient matrix $\operatorname{mat}(\Phi)$ for symmetric $2 n$-variable polynomial matrices $\Phi(\zeta, \eta)$ for general $n>1$, we first need to introduce an ordering on the monomials $\zeta_{1}^{k_{1}} \zeta_{2}^{k_{2}} \cdots \zeta_{n}^{k_{n}}$, equivalently, on the n-tuples of degrees 
$\left(k_{1}, k_{2}, \ldots, k_{n}\right)$. Many orderings are possible, here we choose the anti-lexicographic ordering, which is defined as follows:

Given $\left(a_{1}, a_{2}, \ldots, a_{n}\right),\left(b_{1}, b_{2}, \ldots, b_{n}\right) \in \mathbb{N}^{n}$ we define: $\left(a_{1}, a_{2}, \ldots, a_{n}\right)<\left(b_{1}, b_{2}, \ldots, b_{n}\right)$ if and only if the rightmost nonzero entry of $\left(a_{1}-b_{1}, a_{2}-b_{2}, \ldots, a_{n}-b_{n}\right)$ is $<0$.

As an example, let $n=2$, and consider the set of all 2-tuples $\left(a_{1}, a_{2}\right)$ with $0 \leq a_{1} \leq$ $N_{1}, 0 \leq a_{2} \leq N_{2}$ with $N_{1}, N_{2}$ positive integers. The 2-tuples in this set are ordered as follows:

$$
\begin{array}{ccccc}
(0,0)< & (1,0)< & (2,0)< & \ldots & <\left(N_{1}, 0\right) \\
<(0,1)< & (1,1)< & (2,1)< & \ldots & <\left(N_{1}, 1\right) \\
\vdots & \vdots & \vdots & \ldots & \vdots \\
<\left(0, N_{2}\right)< & \left(1, N_{2}\right)< & \left(2, N_{2}\right)< & \ldots & <\left(N_{1}, N_{2}\right) .
\end{array}
$$

Using the above anti-lexicographic ordering, for a given symmetric $\Phi(\zeta, \eta) \in \mathbb{R}_{s}^{q \times q}[\zeta, \eta]$ a unique coefficient matrix mat $(\Phi)$ is defined, and $\operatorname{mat}(\Phi)$ is symmetric. The matrix mat $(\Phi)$ has as block entries the coefficients $\Phi_{k, l}$, with $k$ and $l$ ordered according to the given ordering. We will explain this now in more detail for $n=2$. Let

$$
\Phi(\zeta, \eta)=\sum_{k_{1}, l_{1}=0}^{N_{1}} \sum_{k_{2}, l_{2}=0}^{N_{2}} \Phi_{\left(k_{1}, k_{2}\right)\left(l_{1}, l_{2}\right)} \zeta_{1}^{k_{1}} \zeta_{2}^{k_{2}} \eta_{1}^{l_{1}} \eta_{1}^{l_{2}} .
$$

Define $V\left(\zeta_{1}\right)=\left(I, \zeta_{1} I, \ldots, \zeta_{1}^{N_{1}} I\right)$, with $I$ the $q \times q$ identity matrix. Then $\Phi(\zeta, \eta)$ can be written as

$$
\Phi(\zeta, \eta)=\left(V\left(\zeta_{1}\right), \zeta_{2} V\left(\zeta_{1}\right), . ., \zeta_{2}^{N_{2}} V\left(\zeta_{1}\right)\right)\left(\begin{array}{cccc}
\Phi^{(0,0)} & \Phi^{(0,1)} & \ldots & \Phi^{\left(0, N_{2}\right)} \\
\Phi^{(1,0)} & \Phi^{(1,1)} & \ldots & \Phi^{\left(1, N_{2}\right)} \\
\vdots & \vdots & \ddots & \vdots \\
\Phi^{\left(N_{2}, 0\right)} & \Phi^{\left(N_{2}, 1\right)} & \ldots & \Phi^{\left(N_{2}, N_{2}\right)}
\end{array}\right)\left(\begin{array}{c}
V\left(\eta_{1}\right) \\
\eta V\left(\eta_{1}\right) \\
\vdots \\
\eta^{N_{2}} V\left(\eta_{1}\right)
\end{array}\right)
$$

where the $\left(N_{1}+1\right) q \times\left(N_{1}+1\right) q$ matrices $\Phi^{(i, j)}$ are defined by

$$
\Phi^{(i, j)}:=\left(\begin{array}{cccc}
\Phi_{(0, i)(0, j)} & \Phi_{(0, i)(1, j)} & \ldots & \Phi_{(0, i)\left(N_{1}, j\right)} \\
\Phi_{(1, i)(0, j)} & \Phi_{(1, i)(1, j)} & \ldots & \Phi_{(1, i)\left(N_{1}, j\right)} \\
\vdots & \vdots & \ddots & \vdots \\
\Phi_{\left(N_{1}, i\right)(0, j)} & \Phi_{\left(N_{1}, i\right)(1, j)} & \ldots & \Phi_{\left(N_{1}, i\right)\left(N_{1}, j\right)}
\end{array}\right) .
$$

Note that $\Phi^{(i, j)}$ has as block entries the coefficient matrices $\Phi_{\left(k_{1}, i\right)\left(l_{1}, j\right)}$ of $\Phi(\zeta, \eta)$ with multi-indices $\left(k_{1}, i\right)$ and $\left(l_{1}, j\right)$ ordered in the given ordering. The matrix in the middle of (4) is the coefficient matrix mat $(\Phi)$ of $\Phi(\zeta, \eta)$. We will denote $\left(V\left(\zeta_{1}\right), \zeta_{2} V\left(\zeta_{1}\right), \ldots, \zeta_{2}^{N_{2}} V\left(\zeta_{1}\right)\right)$ by $V_{2}\left(\zeta_{1}, \zeta_{2}\right)$. Observe that

$$
V_{2}\left(\zeta_{1}, \zeta_{2}\right)=\left(I, \zeta_{1} I, \ldots, \zeta_{1}^{N_{1}} I \vdots \zeta_{2} I, \zeta_{2} \zeta_{1} I, . ., \zeta_{2} \zeta_{1}^{N_{1}} I \vdots \ldots \zeta_{2}^{N_{2}} I, \zeta_{2}^{N_{2}} \zeta_{1} I, \ldots, \zeta_{2}^{N_{2}} \zeta_{1}^{N_{1}} I\right)
$$

and that the monomials $\zeta_{1}^{k_{1}} \zeta_{2}^{k_{2}}$ appearing in this matrix from the left to the right are indeed ordered according to the given ordering. As shorthand notation, we will sometimes write $\operatorname{mat}(\Phi)=\left\{\Phi^{(i, j)}\right\}_{i, j=0,1, \ldots, N_{2}}$. 
Of course, in the same way we can define the coefficient matrix of a given $n$-variable polynomial matrix $F(\xi)$. If $F(\xi)=\Sigma_{k} F_{k} \xi^{k}$, then the anti-lexicographic ordering on the multi-indices $k \in \mathbb{N}^{n}$ uniquely defines the coefficient matrix of $F(\xi)$, which will be denoted by $\operatorname{mat}(F)$.

For a given $2 n$-variable polynomial matrix, factorizations of the coefficient matrix will give rise to factorizations of the $2 n$-variable polynomial matrix. Our algorithms will actually reduce the problems of spectral factorization and sum of squares to factorization problems for these coefficient matrices, which are of course constant real matrices. It is important to note that the correspondence between $\mathbb{R}_{s}^{q \times q}[\zeta, \eta]$ and the set of real symmetric matrices is bijective and that the size of mat $(\Phi)$ depends on the highest monomial degree of $\zeta$ and $\eta$ in $\Phi(\zeta, \eta)$.

We will also consider vectors $\Psi \in\left(\mathbb{R}_{s}^{q \times q}[\zeta, \eta]\right)^{n}$, i.e. $n$-tuples of symmetric $2 n$-variable polynomial matrices $\Psi=\left(\Psi_{1}, \ldots, \Psi_{n}\right)$ with $\Psi_{i} \in \mathbb{R}_{s}^{q \times q}[\zeta, \eta]$. In the same way that $\Phi$ induces a QDF, $\Psi$ induces a vector of quadratic differential forms (VQDF), defined as

$$
\begin{aligned}
& Q_{\Psi}: \mathfrak{C}^{\infty}\left(\mathbb{R}^{n}, \mathbb{R}^{q}\right) \longrightarrow\left(\mathfrak{C}^{\infty}\left(\mathbb{R}^{n}, \mathbb{R}\right)\right)^{n} \\
& Q_{\Psi}(w):=\left(\begin{array}{lll}
Q_{\Psi_{1}}(w), & Q_{\Psi_{2}}(w), & \ldots,
\end{array} Q_{\Psi_{n}}(w)\right) .
\end{aligned}
$$

We review the notion of divergence of a VQDF (see [12]). Given a VQDF $Q_{\Psi}$ as above, its divergence is defined as the QDF

$$
\left(\operatorname{div} Q_{\Psi}\right)(w):=\frac{\partial}{\partial x_{1}} Q_{\Psi_{1}}(w)+\ldots+\frac{\partial}{\partial x_{n}} Q_{\Psi_{n}}(w)
$$

for $w \in \mathfrak{C}^{\infty}\left(\mathbb{R}^{n}, \mathbb{R}^{q}\right)$.

It was shown in [12] that the $2 n$-variable polynomial matrix associated with the divergence of a $\operatorname{VQDF} Q_{\Psi}$ is given by the $2 n$-variable polynomial matrix $\dot{\Psi}$ defined by

$$
\dot{\Psi}(\zeta, \eta):=\left(\zeta_{1}+\eta_{1}\right) \Psi_{1}(\zeta, \eta)+\ldots+\left(\zeta_{n}+\eta_{n}\right) \Psi_{n}(\zeta, \eta) .
$$

In this paper we are interested in $\operatorname{mat}(\dot{\Psi})$, i.e., in the underlying real symmetric coefficient matrix. For the sake of simplicity consider again the case $n=2$. First, for $i=1,2$, define the $i$-block right-shift and $i$-block downward-shift operators, $\sigma_{i, R}$ and $\sigma_{i, D}$ respectively, as follows: with mat $(\Phi)=\left\{\Phi^{(i, j)}\right\}_{i, j=0,1, \ldots, N_{2}}$ we define

$$
\begin{aligned}
& \sigma_{1, R}(\operatorname{mat}(\Phi))=\left\{\left(\begin{array}{cc}
0 & \Phi^{(i, j)} \\
0_{q} & 0
\end{array}\right)\right\}_{i, j=0,1, \ldots, N_{2}} \\
& \sigma_{1, D}(\operatorname{mat}(\Phi))=\left\{\left(\begin{array}{cc}
0 & 0_{q} \\
\Phi^{(i, j)} & 0
\end{array}\right)\right\}_{i, j=0,1, \ldots, N_{2}} \\
& \sigma_{2, R}(\operatorname{mat}(\Phi))=\left(\begin{array}{cc}
0 & \operatorname{mat}(\Phi) \\
0_{\left(N_{1}+1\right) q} & 0
\end{array}\right), \sigma_{2, D}(\operatorname{mat}(\Phi))=\left(\begin{array}{cc}
0 & 0_{\left(N_{1}+1\right) q} \\
\operatorname{mat}(\Phi) & 0
\end{array}\right) .
\end{aligned}
$$

Here $0_{q}$ denotes the $q \times q$ zero matrix, and $0_{\left(N_{1}+1\right) q}$ the $\left(N_{1}+1\right) q \times\left(N_{1}+1\right) q$ zero matrix. The sizes of the other 0-matrices appearing in the above should be apparent from the context. It is straightforward to verify that $\operatorname{mat}\left(\left(\zeta_{1}+\eta_{1}\right) \Phi(\zeta, \eta)\right)=\left(\sigma_{1, D}+\sigma_{1, R}\right)(\operatorname{mat}(\Phi))$ and $\operatorname{mat}\left(\left(\zeta_{2}+\eta_{2}\right) \Phi(\zeta, \eta)\right)=\left(\sigma_{2, D}+\sigma_{2, R}\right)(\operatorname{mat}(\Phi))$. Thus, if $\Psi=\left(\Psi_{1}, \Psi_{2}\right)$, we find that

$$
\operatorname{mat}(\dot{\Psi})=\left(\sigma_{1, D}+\sigma_{1, R}\right)\left(\operatorname{mat}\left(\Psi_{1}\right)\right)+\left(\sigma_{2, D}+\sigma_{2, R}\right)\left(\operatorname{mat}\left(\Psi_{2}\right)\right) .
$$


We now explain how this generalizes to general $n>1$. Let $\Phi$ be a symmetric $2 n$ variable polynomial matrix with highest monomial degree $\left(N_{1}, N_{2}, \ldots, N_{n}\right)$. In order to express $\Phi(\zeta, \eta)$ in terms of its coefficient matrix using monomials in the lexicographic ordering, we define $V_{1}\left(\zeta_{1}\right):=V\left(\zeta_{1}\right)$, and in addition to $V_{2}\left(\zeta_{1}, \zeta_{2}\right)$ we define recursively for $k=2,3, \ldots, n$ :

$V_{k}\left(\zeta_{1}, \zeta_{2}, . ., \zeta_{k}\right):=\left(V_{k-1}\left(\zeta_{1}, \zeta_{2}, . ., \zeta_{k-1}\right) \vdots \zeta_{k} V_{k-1}\left(\zeta_{1}, \zeta_{2}, . ., \zeta_{k-1}\right) \vdots \ldots \vdots \zeta_{k}^{N_{k}} V_{k-1}\left(\zeta_{1}, \zeta_{2}, . ., \zeta_{k-1}\right)\right)$.

The monomials appearing from left to right in the matrix $V_{n}\left(\zeta_{1}, \zeta_{2}, \ldots, \zeta_{n}\right)$ are then ordered in the anti-lexicographic ordering, and therefore we have

$$
\Phi(\zeta, \eta)=V_{n}\left(\zeta_{1}, \zeta_{2}, \ldots, \zeta_{n}\right) \operatorname{mat}(\Phi) V_{n}\left(\eta_{1}, \eta_{2}, \ldots, \eta_{n}\right)^{T} .
$$

In this way we can see that multiplication of $\Phi(\zeta, \eta)$ by $\zeta_{k}$ and $\eta_{k}$ corresponds to appropriate downward-shift and right-shift of blocks within the coefficient matrix mat $(\Phi)$, respectively. These shift operators are called the $k$-block downward-shift and $k$-block right-shift operators, $\sigma_{k, D}$ and $\sigma_{k, R}(k=1,2 \ldots, n)$, respectively. Thus, for $\Psi=\left(\Psi_{1}, \Psi_{2}, \ldots, \Psi_{n}\right)$, we obtain:

$$
\operatorname{mat}(\dot{\Psi})=\left(\sigma_{1, D}+\sigma_{1, R}\right)\left(\operatorname{mat}\left(\Psi_{1}\right)\right)+\left(\sigma_{2, D}+\sigma_{2, R}\right)\left(\operatorname{mat}\left(\Psi_{2}\right)\right)+\cdots+\left(\sigma_{n, D}+\sigma_{n, R}\right)\left(\operatorname{mat}\left(\Psi_{n}\right)\right) .
$$

We conclude this section by noting that the notion of divergence of a VQDF can be given a physical interpretation in the context of dissipative nD systems (see [12]). Indeed, if $\mathfrak{B}$ is a controllable $n \mathrm{D}$ behavior with image representation $\mathfrak{B}=\operatorname{im}(M)$, and $\Phi \in \mathbb{R}_{s}^{q \times q}[\zeta, \eta]$, then the $\operatorname{VQDF} Q_{\Psi}$ with $\Psi \in\left(\mathbb{R}_{s}^{q \times q}[\zeta, \eta]\right)^{n}$ is said to be a storage function for $\mathfrak{B}$ with respect $Q_{\Phi}$ if

$$
\left(\operatorname{div} Q_{\Psi}\right)(\ell) \leq Q_{\Phi}(w)
$$

for all $\ell \in \mathfrak{C}^{\infty}\left(\mathbb{R}^{n}, \mathbb{R}^{q}\right)$ and $w=M\left(\frac{d}{d \mathbf{x}}\right) \ell$. If such storage function exists, then $\mathfrak{B}$ is called dissipative with respect to $Q_{\Phi}$. If the first coordinate $x_{1}$ designates time $t$, and the remaining $x_{2}, \ldots, x_{n}$ are space coordinates, then the inequality (6) can be rewritten as

$$
\frac{\partial}{\partial t} Q_{\Psi_{1}}(\ell) \leq Q_{\Phi}\left(M\left(\frac{d}{d x}\right) \ell\right)-\left[\frac{\partial}{\partial x_{2}} Q_{\Psi_{2}}(\ell)+\frac{\partial}{\partial x_{3}} Q_{\Psi_{3}}(\ell)+\ldots+\frac{\partial}{\partial x_{n}} Q_{\Psi_{n}}(\ell)\right] .
$$

This can be interpreted as saying that the change in stored energy $\frac{\partial}{\partial t} Q_{\Psi_{1}}(\ell)$ in an infinitesimal volume does not exceed the difference between the supply $Q_{\Phi}\left(M\left(\frac{d}{d x}\right) \ell\right)$ into the infinitesimal volume and the energy lost by the volume due to energy flux flowing out of the volume in the directions $x_{2}, \ldots, x_{n}$. The difference between the right hand side and the left hand side is the rate at which energy is dissipated within the volume. This difference is called the dissipation rate.

\section{Lifting $n$ variable to $2 n$ variable polynomial matrices}

In the section following the present one, we show how the problem of multi-dimensional spectral factorization can be reduced to factorization of a real symmetric constant matrices. This result will be achieved by lifting the spectral factorization problem to a $2 n$-variable polynomial context, i.e., by associating with the to-be-factored $n$-variable 
polynomial matrix $Z(\xi)$ a suitable $2 n$-variable polynomial matrix. Lifting the problem to a $2 n$-variable polynomial matrix context allows us to formulate the problem of spectral factorization in terms of the related coefficient matrices. In the present section we will discuss this lifting procedure.

Let $Z(\xi)$ be a real para-hermitian $n$-variable polynomial matrix, $Z(\xi)=\sum_{k} Z_{k} \xi^{k}$ with $Z_{k} \in \mathbb{R}^{q \times q}$. Note that $Z(\xi)$ is para-hermitian if and only if the coefficient matrices $Z_{k}$ satisfy

$$
Z_{\left(k_{1}, k_{2}, \ldots, k_{n}\right)}^{T}=(-1)^{k_{1}+k_{2}+\ldots+k_{n}} Z_{\left(k_{1}, k_{2}, \ldots, k_{n}\right)}
$$

for all multi-indices $\left(k_{1}, k_{2}, \ldots, k_{n}\right)$. In the following, we will associate with $Z(\xi)$ symmetric, $2 n$-variable polynomial matrices $\Phi(\zeta, \eta)$ with the property that

$$
\Phi(-\xi, \xi)=Z(\xi) .
$$

For a given $Z(\xi)$ there are always infinitely many $\Phi$ 's such that this property holds. For example, whenever $\Phi$ satisfies (7) then also $\Phi^{\prime}(\zeta, \eta):=\Phi(\zeta, \eta)+\left(\zeta_{1}+\eta_{1}\right) \Gamma_{1}(\zeta, \eta)+\left(\zeta_{2}+\right.$ $\left.\eta_{2}\right) \Gamma_{2}(\zeta, \eta)+\ldots+\left(\zeta_{n}+\eta_{n}\right) \Gamma_{n}(\zeta, \eta)$ satisfies $(7)$, for any choice of $\Gamma_{1}, \ldots, \Gamma_{n} \in \mathbb{R}_{s}^{q \times q}[\zeta, \eta]$. One possible choice of $\Phi$ such that (7) holds is given by

$$
\Phi(\zeta, \eta):=\frac{1}{2}\left(Z^{T}(\zeta)+Z(\eta)\right)
$$

Since $\Phi(\eta, \zeta)^{T}=\frac{1}{2}\left(Z(\eta)+Z^{T}(\zeta)\right)=\Phi(\zeta, \eta), \Phi(\zeta, \eta)$ is indeed symmetric. Using $Z^{T}(-\xi)=$ $Z(\xi)$, $\Phi$ satisfies $(7)$. Note that for this particular choice of $\Phi(\zeta, \eta)$, the highest monomial degree of $\zeta$ and $\eta$ occurring in the entries of $\Phi(\zeta, \eta)$ is equal to the highest monomial degree occurring in $Z(\xi)$. This is not true for all choices of $\Phi(\zeta, \eta)$.

In the following, we will establish a characterization of all symmetric $2 n$-variable polynomial matrices $\Phi(\zeta, \eta)$ such that $\Phi(-\xi, \xi)=Z(\xi)$. For the special case that $n=1$ this was done in [15]. In fact, there the following was proven:

Proposition 3.1 Let $Z(\xi)$ be a para-hermitian 1-variable polynomial matrix, $Z(\xi)=$ $Z_{0}+Z_{1} \xi+Z_{2} \xi^{2}+\ldots Z_{M} \xi^{M}$, with $Z_{M} \neq 0$. Let $\Phi(\zeta, \eta)=\sum_{k, j} \Phi_{k, j} \zeta^{k} \eta^{j}$ be a symmetric two-variable polynomial matrix. Then $\Phi(-\xi, \xi)=Z(\xi)$ if and only if $\Phi_{0, k}-\Phi_{1, k-1}+$ $\Phi_{2, k-2}-\ldots+(-1)^{k} \Phi_{k, 0}=Z_{k}$ for all $k=0,1, \ldots, M$.

In terms of the coefficient matrix mat $(\Phi)$, this requires that the $M+1$ anti-diagonals, with an appropriate sign-pattern, add up to the coefficients of $Z(\xi)$.

In the present paper, we will concentrate first on the case $n=2$, and comment later on how to generalize this to the general case $n>2$. Let a para-hermitian 2-variable polynomial matrix $Z\left(\xi_{1}, \xi_{2}\right)$ be given by

$$
Z\left(\xi_{1}, \xi_{2}\right)=\sum_{k=0}^{M_{1}} \sum_{l=0}^{M_{2}} Z_{k, l} \xi_{1}^{k} \xi_{2}^{l} .
$$

On the other hand, let $\Phi\left(\zeta_{1}, \zeta_{2}, \eta_{1}, \eta_{2}\right)$ be a symmetric 4 -variable polynomial matrix given by

$$
\Phi\left(\zeta_{1}, \zeta_{2}, \eta_{1}, \eta_{2}\right)=\sum_{k_{1}, l_{1}=0}^{N_{1}} \sum_{k_{2}, l_{2}=0}^{N_{2}} \Phi_{\left(k_{1}, k_{2}\right)\left(l_{1}, l_{2}\right)} \zeta_{1}^{k_{1}} \zeta_{2}^{k_{2}} \eta_{1}^{l_{1}} \eta_{2}^{l_{2}}
$$


Clearly,

$$
\Phi\left(-\xi_{1},-\xi_{2}, \xi_{1}, \xi_{2}\right)=\sum_{k_{1}, l_{1}=0}^{N_{1}} \sum_{k_{2}, l_{2}=0}^{N_{2}} \Phi_{\left(k_{1}, k_{2}\right)\left(l_{1}, l_{2}\right)}(-1)^{k_{1}+k_{2}} \xi_{1}^{k_{1}+l_{1}} \xi_{2}^{k_{2}+l_{2}} .
$$

Thus, a necessary condition for $\Phi(-\xi, \xi)=Z(\xi)$ is that $2 N_{1} \geq M_{1}$ and $2 N_{2} \geq M_{2}$. Therefore, for notational convenience, we will write

$$
Z\left(\xi_{1}, \xi_{2}\right)=\sum_{k=0}^{2 N_{1}} \sum_{l=0}^{2 N_{2}} Z_{k, l} \xi_{1}^{k} \xi_{2}^{l}
$$

where we define $Z_{k, l}:=0$ for $k=M_{1}+1, \ldots, 2 N_{1}$ or $l=M_{2}+1, \ldots, 2 N_{2}$. We will describe now the relations between the $Z_{k, l}$ and the $\Phi_{\left(k_{1}, k_{2}\right)\left(l_{1}, l_{2}\right)}$ that are necessary and sufficient for $\Phi(-\xi, \xi)=Z(\xi)$. Recall

$$
\operatorname{mat}(\Phi)=\left(\begin{array}{cccc}
\Phi^{(0,0)} & \Phi^{(0,1)} & \ldots & \Phi^{\left(0, N_{2}\right)} \\
\Phi^{(1,0)} & \Phi^{(1,1)} & \ldots & \Phi^{\left(1, N_{2}\right)} \\
\vdots & \vdots & \ddots & \vdots \\
\Phi^{\left(N_{2}, 0\right)} & \Phi^{\left(N_{2}, 1\right)} & \ldots & \Phi^{\left(N_{2}, N_{2}\right)}
\end{array}\right),
$$

where for $i, j=0,1, \ldots, N_{1}$ we have

$$
\Phi^{(i, j)}:=\left(\begin{array}{cccc}
\Phi_{(0, i)(0, j)} & \Phi_{(0, i)(1, j)} & \ldots & \Phi_{(0, i)\left(N_{1}, j\right)} \\
\Phi_{(1, i)(0, j)} & \Phi_{(1, i)(1, j)} & \ldots & \Phi_{(1, i)\left(N_{1}, j\right)} \\
\vdots & \vdots & \ddots & \vdots \\
\Phi_{\left(N_{1}, i\right)(0, j)} & \Phi_{\left(N_{1}, i\right)(1, j)} & \ldots & \Phi_{\left(N_{1}, i\right)\left(N_{1}, j\right)}
\end{array}\right) .
$$

We first consider the $Z_{k, 0}, k=0,1, \ldots, 2 N_{1}$. It follows from (8) that these coefficient matrices are determined by the block $\Phi^{(0,0)}$, in particular by taking the sum of the blocks on the $2 N_{1}+1$ anti-diagonals of this matrix, with an appropriate sign pattern. Note that

$$
\Phi^{(0,0)}:=\left(\begin{array}{cccc}
\Phi_{(0,0)(0,0)} & \Phi_{(0,0)(1,0)} & \ldots & \Phi_{(0,0)\left(N_{1}, 0\right)} \\
\Phi_{(1,0)(0,0)} & \Phi_{(1,0)(1,0)} & \ldots & \Phi_{(1,0)\left(N_{1}, 0\right)} \\
\vdots & \vdots & \ddots & \vdots \\
\Phi_{\left(N_{1}, 0\right)(0,0)} & \Phi_{\left(N_{1}, 0\right)(1,0)} & \ldots & \Phi_{\left(N_{1}, 0\right)\left(N_{1}, 0\right)}
\end{array}\right) .
$$

The conditions are:

$$
\begin{aligned}
Z_{k, 0}= & \Phi_{(0,0)(k, 0)}-\Phi_{(1,0)(k-1,0)}+\ldots+(-1)^{k-1} \Phi_{(k-1,0)(1,0)}+(-1)^{k} \Phi_{(k, 0)(0,0)} \\
& \left(k=0,1, \ldots, N_{1}\right) \\
Z_{N_{1}+k, 0}= & (-1)^{k} \Phi_{(k, 0)\left(N_{1}, 0\right)}+(-1)^{k+1} \Phi_{(k+1,0)\left(N_{1}-1,0\right)}+\ldots+(-1)^{N_{1}-1} \Phi_{\left(N_{1}-1,0\right)(k+1,0)} \\
& +(-1)^{N_{1}} \Phi_{\left(N_{1}, 0\right)(k, 0)} \\
& \left(k=1,2, \ldots, N_{1}\right) .
\end{aligned}
$$

In particular, $Z_{0,0}=\Phi_{(0,0)(0,0)}, Z_{1,0}=\Phi_{(0,0)(1,0)}-\Phi_{(1,0)(0,0)}, Z_{2,0}=\Phi_{(0,0)(2,0)}-\Phi_{(1,0)(1,0)}+$ $\Phi_{(2,0)(0,0)}$ up to, finally, $Z_{2 N_{1}, 0}=(-1)^{N_{1}} \Phi_{\left(N_{1}, 0\right)\left(N_{1}, 0\right)}$.

Next, we consider the $Z_{k, l}, k=0,1, \ldots, 2 N_{1}$, first for $l=1,2, \ldots, N_{2}$. These turn out to be determined by the anti-diagonal blocks $\Phi^{(0, l)}, \Phi^{(1, l-1)}, \Phi^{(2, l-2)}, \ldots \Phi^{(l-1,1)}, \Phi^{(l, 0)}$ of 
mat $(\Phi)$. The easiest way to visualize the conditions are by forming a matrix $\Omega_{l}$ defined by

$$
\Omega_{l}:=\left(\begin{array}{ccccc}
0 & 0 & \ldots & 0 & \Phi^{(0, l)} \\
0 & 0 & \ldots & \Phi^{(1, l-1)} & 0 \\
\vdots & & & \vdots & \vdots \\
\Phi^{(l, 0)} & 0 & 0 & 0 & 0
\end{array}\right)
$$

with blocks $\Phi^{(i, l-i)}$ on the main anti-diagonal, and zeroes elsewehere. Starting in the upper right corner at $\Phi^{(0, l)}$, going down to the lower left corner at $\Phi^{(l, 0)}$ one can form $2 N_{1}+1$ anti-diagonals with blocks $\Phi_{\left(k_{1}, k_{2}\right)\left(l_{1}, l_{2}\right)}$. By taking the sum of the blocks on the $k$ th anti-diagonal, with appropriate sign pattern, one obtains the $Z_{k, l}$, for $k=0,1, \ldots, 2 N_{1}$. The sign of each term is determined by $(-1)^{k_{1}+k_{2}}$.

For example, $Z_{k, 1}\left(k=0,1, \ldots, 2 N_{1}\right)$ is determined by

$$
\Omega_{1}=\left(\begin{array}{cc}
0 & \Phi^{(0,1)} \\
\Phi^{(1,0)} & 0
\end{array}\right)
$$

Indeed, $Z_{0,1}=\Phi_{(0,0)(0,1)}-\Phi_{(0,1)(0,0)}, Z_{11}=\Phi_{(0,0)(1,1)}-\Phi_{(1,0)(0,1)}-\Phi_{(0,1)(1,0)}+\Phi_{(1,1)(0,0)}$, etc.

Finally we consider the $Z_{k, N_{2}+l}, k=0,1, \ldots, 2 N_{1}$, for $l=1,2, \ldots, N_{2}$. These are determined by the anti-diagonal blocks $\Phi^{\left(l, N_{2}\right)}, \Phi^{\left(l+1, N_{2}-1\right)}, \Phi^{\left(l+2, N_{2}-2\right)}, \ldots \Phi^{\left(N_{2}-1, l+1\right)}, \Phi^{\left(N_{2}, l\right)}$ of $\operatorname{mat}(\Phi)$. We form a matrix $\Omega_{l}^{\prime}$ defined by

$$
\Omega_{l}^{\prime}:=\left(\begin{array}{ccccc}
0 & 0 & \ldots & 0 & \Phi^{\left(l, N_{2}\right)} \\
0 & 0 & \ldots & \Phi^{\left(l+1, N_{2}-1\right)} & 0 \\
\vdots & & & \vdots & \vdots \\
\Phi^{\left(N_{2}, l\right)} & 0 & 0 & 0 & 0
\end{array}\right)
$$

with blocks $\Phi^{\left(l+i, N_{2}-i\right)}$ on the main anti-diagonal, and zeroes elsewehere. Starting in the upper right corner at $\Phi^{\left(l, N_{2}\right)}$, going down to the lower left corner at $\Phi^{\left(N_{2}, l\right)}$ one can form $2 N_{1}+1$ anti-diagonals with blocks $\Phi_{\left(k_{1}, k_{2}\right)\left(l_{1}, l_{2}\right)}$. Again by taking the sum of the blocks on the $k$ th anti-diagonal, with appropriate sign pattern, one obtains the $Z_{k, N_{2}+l}$, for $k=0,1, \ldots, 2 N_{1}$. The sign of each term is determined by $(-1)^{k_{1}+k_{2}}$. For example, $Z_{2 N_{1}, 2 N_{2}}$, determined by $\Phi^{\left(N_{1}, N_{2}\right)}$, is equal to $(-1)^{N_{1}+N_{2}} \Phi_{\left(N_{1}, N_{2}\right)\left(N_{1}, N_{2}\right)}$.

Example 3.1 As a simple example, consider the case that $q=1$ and $Z\left(\xi_{1}, \xi_{2}\right)$ is the scalar 2-variable polynomial given by $Z\left(\xi_{1}, \xi_{2}\right)=\xi_{1}^{2} \xi_{2}^{2}+2 \xi_{1} \xi_{2}-\xi_{1}^{2}+1$. By the above considerations, it is possible to find a scalar 4 -variable polynomial matrix $\Phi\left(\zeta_{1}, \zeta_{2}, \eta_{1}, \eta_{2}\right)$ with highest monomial degree $\left(N_{1}, N_{2}\right)=(1,1)$. The (unknown) coefficient matrix mat $(\Phi)$ is therefore a symmetric $4 \times 4$ matrix:

$$
\operatorname{mat}(\Phi)=\left(\begin{array}{ll|ll}
\Phi_{(0,0)(0,0)} & \Phi_{(0,0)(1,0)} & \Phi_{(0,0)(0,1)} & \Phi_{(0,0)(1,1)} \\
\Phi_{(1,0)(0,0)} & \Phi_{(1,0)(1,0)} & \Phi_{(1,0)(0,1)} & \Phi_{(1,0)(1,1)} \\
\hline \Phi_{(0,1)(0,0)} & \Phi_{(0,1)(1,0)} & \Phi_{(0,1)(0,1)} & \Phi_{(0,1)(1,1)} \\
\Phi_{(1,1)(0,0)} & \Phi_{(1,1)(1,0)} & \Phi_{(1,1)(0,1)} & \Phi_{(1,1)(1,1)}
\end{array}\right) .
$$


The conditions for $Z(\xi)=\Phi(-\xi, \xi)$ then become:

$$
\begin{aligned}
Z_{0,0} & =\Phi_{(0,0)(0,0)} \\
Z_{1,0} & =\Phi_{(0,0)(1,0)}-\Phi_{(1,0)(0,0)} \\
Z_{2,0} & =-\Phi_{(1,0)(1,0)} \\
Z_{0,1} & =\Phi_{(0,0)(0,1)}-\Phi_{(0,1)(0,0)} \\
Z_{1,1} & =\Phi_{(0,0)(1,1)}-\Phi_{(1,0)(0,1)}-\Phi_{(0,1)(1,0)}+\Phi_{(1,1)(0,0)} \\
Z_{2,1} & =-\Phi_{(1,0)(1,1)}+\Phi_{(1,1)(1,0)} \\
Z_{0,2} & =-\Phi_{(0,1)(0,1)} \\
Z_{1,2} & =-\Phi_{(0,1)(1,1)}+\Phi_{(1,1)(0,1)} \\
Z_{2,2} & =\Phi_{(1,1)(1,1)}
\end{aligned}
$$

In our example, $Z_{0,0}=1, Z_{2,0}=-1, Z_{1,1}=2, Z_{2,2}=1$, and the remaining $Z_{k, l}$ are 0 . One particular choice of mat $(\Phi)$ is therefore given by

$$
\operatorname{mat}(\Phi)=\left(\begin{array}{cccc}
1 & 0 & 0 & 1 \\
0 & 1 & 0 & 1 \\
0 & 0 & 0 & 2 \\
1 & 1 & 2 & 1
\end{array}\right)
$$

Pre-and post multiplying with $\left(1, \zeta_{1}, \zeta_{2}, \zeta_{1} \zeta_{2}\right)$ and $\left(1, \eta_{1}, \eta_{2}, \eta_{1} \eta_{2}\right)^{T}$ yields the corresponding $\Phi\left(\zeta_{1}, \zeta_{2}, \eta_{1}, \eta_{2}\right)$.

In [15], for a given para-hermitian 1-variable polynomial matrix $Z(\xi)$, an explicit formula for a minimal degree symmetric $\Phi(\zeta, \eta)$ satisfying $\Phi(-\xi, \xi)=Z(\xi)$ was obtained. In fact, if $Z(\xi)=Z_{0}+Z_{1} \xi+Z_{2} \xi^{2}+\ldots Z_{M} \xi^{M}$, with $Z_{M} \neq 0$, and $M$ even, then a 2 -variable polynomial matrix of minimal degree is given in terms of its coefficient matrix by

$$
\operatorname{mat}(\Phi)=\frac{1}{2}\left(\begin{array}{cccccc}
2 Z_{0} & Z_{1} & Z_{2} & \ldots & Z_{\frac{M}{2}-1} & Z_{\frac{M}{2}} \\
Z_{1}^{T} & 0 & 0 & \ldots & 0 & -Z_{\frac{M}{2}+1} \\
Z_{2}^{T} & 0 & 0 & \ldots & 0 & Z_{\frac{M}{2}+2} \\
\vdots & \vdots & \vdots & \ddots & \vdots & \vdots \\
Z_{\frac{M}{2}-1}^{T} & 0 & 0 & \ldots & 0 & (-1)^{\frac{M}{2}-1} Z_{M-1} \\
Z_{\frac{M}{2}}^{T} & -Z_{\frac{M}{2}+1}^{T} & Z_{\frac{M}{2}+2}^{T} & \ldots & (-1)^{\frac{M}{2}-1} Z_{M-1}^{T} & 2(-1)^{\frac{M}{2}} Z_{M}
\end{array}\right)
$$

Note that the two-variable polynomial matrix $\Phi$ defined in this way has degree $\frac{M}{2}$. A related $\Phi$, with degree $\frac{M+1}{2}$, can be defined for the case that $M$ is odd.

We will now extend this result to the case $n=2$. Let $Z(\xi)$ be a para-hermitian 2-variable $q \times q$ polynomial matrix, $Z\left(\xi_{1}, \xi_{2}\right)=\sum_{k=0}^{M_{1}} \sum_{l=0}^{M_{2}} Z_{k, l} \xi_{1}^{k} \xi_{2}^{l}$, with $M_{1}, M_{2}$ both even. For $j=0,1,2 \ldots M_{2}$, define

$$
Z_{j}:=\left(\begin{array}{ccccc}
Z_{0, j} & Z_{1, j} & \ldots & Z_{\frac{M_{1}}{2}-1, j} & Z_{\frac{M_{1}}{2}, j} \\
0 & 0 & \ldots & 0 & -Z_{\frac{M_{1}}{2}+1, j} \\
0 & 0 & \ldots & 0 & Z_{\frac{M_{1}}{2}+2, j} \\
\vdots & \vdots & \ddots & \vdots & \vdots \\
0 & 0 & \ldots & 0 & (-1)^{\frac{M_{1}}{2}} Z_{M_{1}, j}
\end{array}\right),
$$


Also define

$$
\begin{aligned}
\Phi^{(0,0)} & :=\frac{1}{2}\left(Z_{0}+Z_{0}^{T}\right), \\
\Phi^{(0, j)} & :=\frac{1}{2} Z_{j}, \quad j=1,2, \ldots, \frac{M_{2}}{2}, \\
\Phi^{(j, 0)} & :=\frac{1}{2} Z_{j}^{T}, \quad j=1,2, \ldots, \frac{M_{2}}{2}, \\
\Phi^{\left(i, \frac{M_{2}}{2}\right)} & :=(-1)^{i} Z_{\frac{M_{2}}{2}+i}, \quad i=1,2 \ldots, \frac{M_{2}}{2}, \\
\Phi^{\left(\frac{M_{2}}{2}, i\right)} & :=(-1)^{i} Z_{\frac{M_{2}}{2}+i}^{T}, i=1,2 \ldots, \frac{M_{2}}{2}, \\
\Phi^{\left(\frac{M_{2}}{2}, \frac{M_{2}}{2}\right)} & :=\frac{1}{2}(-1)^{\frac{M_{2}}{2}}\left(Z_{M_{2}}+Z_{M_{2}}^{T}\right)
\end{aligned}
$$

By applying the formulas obtained earlier in this section, one finds that if we take

$$
\operatorname{mat}(\Phi)=\left(\begin{array}{ccccc}
\Phi^{(0,0)} & \Phi^{(0,1)} & \ldots & \Phi^{\left(0, \frac{M_{2}}{2}-1\right)} & \Phi^{\left(0, \frac{M_{2}}{2}\right)} \\
\Phi^{(1,0)} & 0 & \ldots & 0 & \Phi^{\left(1, \frac{M_{2}}{2}\right)} \\
\vdots & \vdots & \ddots & \vdots & \\
\Phi^{\left(\frac{M_{2}}{2}-1,0\right)} & 0 & \ldots & 0 & \Phi^{\left(\frac{M_{2}}{2}-1, \frac{M_{2}}{2}\right)} \\
\Phi^{\left(\frac{M_{2}}{2}, 0\right)} & \Phi^{\left(\frac{M_{2}}{2}, 1\right)} & \ldots & \Phi^{\left(\frac{M_{2}}{2}, \frac{M_{2}}{2}-1\right)} & \Phi^{\left(\frac{M_{2}}{2}, \frac{M_{2}}{2}\right)}
\end{array}\right),
$$

then $\Phi\left(\zeta_{1}, \zeta_{2}, \eta_{1}, \eta_{2}\right)$ satifies $\Phi\left(-\xi_{1},-\xi_{2}, \xi_{1}, \xi_{2}\right)=Z\left(\xi_{1}, \xi_{2}\right)$. The highest monomial degree of $\Phi$ is $\left(\frac{M_{1}}{2}, \frac{M_{2}}{2}\right)$, which corresponds to $\operatorname{mat}(\Phi)$ having size $\left(\frac{M_{1}}{2}+1\right)\left(\frac{M_{2}}{2}+1\right) q$. Clearly, $\operatorname{mat}(\Phi)$ has minimal size over all $\Phi$ such that $\Phi(-\xi, \xi)=Z(\xi)$. In a similar manner, if $M_{1}$ and $M_{2}$ are odd, then $\Phi$ can be found with coefficient matrix of minimal size $\left(\frac{M_{1}+1}{2}+\right.$ 1) $\left(\frac{M_{2}+1}{2}+1\right) q$. If $M_{1}$ is even and $M_{2}$ is odd then the minimal size is $\left(\frac{M_{1}}{2}+1\right)\left(\frac{M_{2}+1}{2}+1\right) q$, and if $M_{1}$ is odd and $M_{2}$ is even the minimal size is $\left(\frac{M_{1}+1}{2}+1\right)\left(\frac{M_{2}}{2}+1\right) q$.

All of the above can be generalized to the general case $n>1$ by applying similar ideas to the more complicated block structure that was described at the end of the previous section. The notation however becomes rather cumbersome. We omit the details here.

\section{Spectral factorization}

In this section we will present algorithms for multidimensional spectral factorization. We distinguish two different spectral factorization problems. In the first one we will search for spectral factors which are polynomial matrices and reduce the problem to a linear matrix inequality. In the second case we will allow the spectral factors to be rational matrices, and connect it to a linear eigenvalue problem, and to a problem of finding a feasible point of a semialgebraic set.

Theorem 4.1 Let $Z(\xi)$ be a $q \times q$ para-hermitian n-variable polynomial matrix. Let $\Phi(\zeta, \eta) \in \mathbb{R}^{q \times q}[\zeta, \eta]$ be any symmetric $2 n$-variable polynomial matrix such that $\Phi(-\xi, \xi)=$ $Z(\xi)$. Then the following statements are equivalent:

(i) $Z(\xi)$ admits a spectral factorization, i.e. there exists a positive integer $r$ and a $r \times q$ $n$-variable polynomial matrix $F(\xi)$ such that

$$
Z(\xi)=F^{T}(-\xi) F(\xi) .
$$


(ii) There exists an $n$-tuple of symmetric $2 n$-variable polynomial matrices $\Psi(\zeta, \eta)=\left(\Psi_{1}(\zeta, \eta), . ., \Psi_{n}(\zeta, \eta)\right)$, with $\Psi_{i} \in \mathbb{R}_{s}^{q \times q}[\zeta, \eta], i=1, \ldots, n$, such that

$$
\operatorname{mat}(\Phi-\dot{\Psi}) \geq 0
$$

(iii) There exists a symmetric $2 n$-variable polynomial matrix $\hat{\Phi}(\zeta, \eta)$ such that $\hat{\Phi}(-\xi, \xi)=$ $Z(\xi)$ and $\operatorname{mat}(\hat{\Phi}) \geq 0$.

Assume that any of these conditions hold. Then an $F$ such that (11) holds can be computed as follows: find $\Psi(\zeta, \eta)=\left(\Psi_{1}(\zeta, \eta), . ., \Psi_{n}(\zeta, \eta)\right)$, such that (12) holds, and factorize

$$
\operatorname{mat}(\Phi-\dot{\Psi})=\tilde{F}^{T} \tilde{F}
$$

where $\tilde{F}$ is a constant matrix with full row rank, say $r$. Then the polynomial matrix $F(\xi)$ with coefficient matrix $\tilde{F}$ (i.e., $\operatorname{mat}(F)=\tilde{F}$ ) satisfies (11).

Proof : $(i) \Rightarrow($ ii $)$ Clearly $\Phi(-\xi, \xi)-F^{T}(-\xi) F(\xi)=0$. By ([12], theorem 4), there exists a vector of $2 n$-variable polynomial matrices $\Psi(\zeta, \eta)=\left(\Psi_{1}(\zeta, \eta), . ., \Psi_{n}(\zeta, \eta)\right)$ with $\Psi_{i} \in \mathbb{R}^{q \times q}[\zeta, \eta]$ such that $\Phi(\zeta, \eta)-F^{T}(\zeta) F(\eta)=\dot{\Psi}(\zeta, \eta)=\left(\zeta_{1}+\eta_{1}\right) \Psi_{1}(\zeta, \eta)+\ldots+\left(\zeta_{n}+\right.$ $\left.\eta_{n}\right) \Psi_{n}(\zeta, \eta)$. This equality can be written, in terms of the associated coefficient matrices as $\operatorname{mat}(\Phi)-\tilde{F}^{T} \tilde{F}=\operatorname{mat}(\dot{\Psi})$, where $\tilde{F}$ is the coefficient matrix of $F(\xi)$. This implies (12) since $\tilde{F}^{T} \tilde{F} \geq 0$.

$(i i) \Rightarrow(i)$ : Since $\operatorname{mat}(\Phi-\dot{\Psi})$ is a symmetric positive semi definite matrix we can factorize it as $\operatorname{mat}(\Phi-\dot{\Psi})=\tilde{F}^{T} \tilde{F}$. Now let $F(\xi)$ be the $n$-variable polynomial matrix with coefficient matrix $\tilde{F}$. Then we obtain $\Phi(\zeta, \eta)-\dot{\Psi}(\zeta, \eta)=F^{T}(\zeta) F(\eta)$. By taking $\zeta=-\xi$ and $\eta=\xi$ we then obtain $Z(\xi)=\Phi(-\xi, \xi)=F^{T}(-\xi) F(\xi)$.

$($ i $) \Rightarrow($ iii $)$ Define $\hat{\Phi}(\zeta, \eta):=F^{T}(\zeta) F(\eta)$.

(iii) $\Rightarrow(i)$ Factor mat $(\hat{\Phi})=\tilde{F}^{T} \tilde{F}$ and define $F(\xi)$ as the $n$-variable polynomial matrix such that $\operatorname{mat}(F)=\tilde{F}$.

Remark 4.1 Condition (ii) above can be reformulated in terms of the underlying QDF's as $\operatorname{div}\left(Q_{\Psi}(w)\right) \leq Q_{\Phi}(w)$ for all $w \in \mathfrak{C}^{\infty}\left(\mathbb{R}^{n}, \mathbb{R}^{q}\right)$, equivalently, the $n \mathrm{D}$ system with behavior $\mathfrak{B}=\mathfrak{C}^{\infty}\left(\mathbb{R}^{n}, \mathbb{R}^{q}\right)$ is dissipative with respect to the supply rate $Q_{\Phi}$, and $Q_{\Psi}$ is a storage function. Thus, the idea of the theorem is to compute a spectral factor $F(\xi)$ of $Z(\xi)$ by means of computing a storage function $Q_{\Psi}$ for the $Q_{\Phi}$-dissipative system $\mathfrak{C}^{\infty}\left(\mathbb{R}^{n}, \mathbb{R}^{q}\right)$. In fact, $\left\|F\left(\frac{d}{d x}\right) w\right\|^{2}$ is the corresponding dissipation rate, see also [12].

The central issue is that the inequality (12) is in fact a linear matrix inequality, and a solution can be computed, for instance, using the LMI Toolbox in Matlab.

In [15], the above theorem was used for spectral factorization of 1-variable polynomial matrices. Here, we will now take a closer look at the case $n=2$. In this case, for a given $\Phi\left(\zeta_{1}, \zeta_{2}, \eta_{1}, \eta_{2}\right)$ with highest monomial degree $\left(N_{1}, N_{2}\right)$, the size of mat $(\Phi)$ is equal to $q\left(N_{1}+1\right)\left(N_{2}+1\right)$, so the sizes of the unknowns mat $\left(\Psi_{1}\right)$ and $\operatorname{mat}\left(\Psi_{2}\right)$ are $q N_{1}\left(N_{2}+1\right)$ and $q\left(N_{1}+1\right) N_{2}$, respectively. The LMI (12) then becomes:

$$
\operatorname{mat}(\Phi)-\sigma_{1, R}\left(\operatorname{mat}\left(\Psi_{1}\right)\right)-\sigma_{1, D}\left(\operatorname{mat}\left(\Psi_{1}\right)\right)-\sigma_{2, R}\left(\operatorname{mat}\left(\Psi_{2}\right)\right)-\sigma_{2, D}\left(\operatorname{mat}\left(\Psi_{2}\right)\right) \geq 0 .
$$


If we partition the unknown mat $\left(\Psi_{1}\right)$ as

$$
\operatorname{mat}\left(\Psi_{1}\right)=\left\{\Psi_{1}^{(i, j)}\right\}_{i, j=0,1, \ldots N_{2}}
$$

with $\Psi_{1}^{(i, j)}$ of size $q N_{1} \times q N_{1}$, then we have

$$
\begin{gathered}
\sigma_{1, R}\left(\operatorname{mat}\left(\Psi_{1}\right)\right)=\left\{\left(\begin{array}{cc}
0 & \Psi_{1}^{(i, j)} \\
0_{q} & 0
\end{array}\right)\right\}_{i, j=0,1, . ., N_{2}} \\
\sigma_{1, D}\left(\operatorname{mat}\left(\Psi_{1}\right)\right)=\left\{\left(\begin{array}{cc}
0 & 0 \\
\Psi_{1}^{(i, j)} & 0
\end{array}\right)\right\}_{i, j=0,1, \ldots, N_{2}} \\
\sigma_{2, R}\left(\operatorname{mat}\left(\Psi_{2}\right)\right)=\left(\begin{array}{cc}
0 & \operatorname{mat}\left(\Psi_{2}\right) \\
0_{\left(N_{1}+1\right) q} & 0
\end{array}\right), \sigma_{2, D}\left(\operatorname{mat}\left(\Psi_{2}\right)\right)=\left(\begin{array}{cc}
0 & 0_{\left(N_{1}+1\right) q} \\
\operatorname{mat}\left(\Psi_{2}\right) & 0
\end{array}\right) .
\end{gathered}
$$

For general $n>2$, the linear matrix inequality (12) involves all $k$-block downward and right-shift operators $\sigma_{k, D}$ and $\sigma_{k, R}(k=1,2, \ldots n)$ and takes the form

$\operatorname{mat}(\Phi)-\left(\sigma_{1, D}+\sigma_{1, R}\right)\left(\operatorname{mat}\left(\Psi_{1}\right)\right)-\left(\sigma_{2, D}+\sigma_{2, R}\right)\left(\operatorname{mat}\left(\Psi_{2}\right)\right)-\ldots-\left(\sigma_{n, D}+\sigma_{n, R}\right)\left(\operatorname{mat}\left(\Psi_{n}\right)\right) \geq 0$.

If $\Phi(\zeta, \eta)$ has highest monomial degree $\left(N_{1}, N_{2}, \ldots, N_{n}\right)$, then mat $\left(\Psi_{i}\right)$ has size $n_{i}:=$ $q N_{i} \prod_{j=1, j \neq i}^{n}\left(N_{j}+1\right)$. Since the $\Psi_{i}$, equivalently the matrices mat $\left(\Psi_{i}\right)$, can always be taken to be symmetric, the actual number of unknowns in the LMI (12) is equal to $\sum_{i=1}^{n} \frac{n_{i}\left(n_{i}+1\right)}{2}$

Example 4.1 We continue with example 3.1 here. Take $\Phi\left(\zeta_{1}, \zeta_{2}, \eta_{1}, \eta_{2}\right)$ with mat $(\Phi)$ given by $(9)$. Since the highest monomial degree $\left(N_{1}, N_{2}\right)=(1,1)$, the unknowns $\operatorname{mat}\left(\Psi_{1}\right)$ and $\operatorname{mat}\left(\Psi_{2}\right)$ both have size $2 \times 2$. Write

$$
\operatorname{mat}\left(\Psi_{1}\right)=\left(\begin{array}{cc}
a & b \\
b & c
\end{array}\right), \quad \operatorname{mat}\left(\Psi_{2}\right)=\left(\begin{array}{cc}
p & q \\
q & r
\end{array}\right) .
$$

We have

$$
\begin{aligned}
\sigma_{1, R}\left(\operatorname{mat}\left(\Psi_{1}\right)\right) & =\left(\begin{array}{cccc}
0 & a & 0 & b \\
0 & 0 & 0 & 0 \\
0 & b & 0 & c \\
0 & 0 & 0 & 0
\end{array}\right), \sigma_{1, D}\left(\operatorname{mat}\left(\Psi_{1}\right)\right)=\left(\begin{array}{cccc}
0 & 0 & 0 & 0 \\
a & 0 & b & 0 \\
0 & 0 & 0 & 0 \\
b & 0 & c & 0
\end{array}\right), \\
\sigma_{2, R}\left(\operatorname{mat}\left(\Psi_{2}\right)\right) & =\left(\begin{array}{cccc}
0 & 0 & p & q \\
0 & 0 & q & r \\
0 & 0 & 0 & 0 \\
0 & 0 & 0 & 0
\end{array}\right), \sigma_{2, D}\left(\operatorname{mat}\left(\Psi_{2}\right)\right)=\left(\begin{array}{llll}
0 & 0 & 0 & 0 \\
0 & 0 & 0 & 0 \\
p & q & 0 & 0 \\
q & r & 0 & 0
\end{array}\right) .
\end{aligned}
$$

Thus the LMI in the unknowns $a, b, c, p, q, r$ becomes

$$
\left(\begin{array}{cccc}
1 & a & p & 1+b+q \\
a & 1 & b+q & 1+r \\
p & b+q & 0 & 2+c \\
1+b+q & 1+r & 2+c & 1+c
\end{array}\right) \geq 0
$$


A solution is obtained by taking $a=1, b, p, q, r=0, c=-2$. Indeed, with these values we obtain

$$
\left(\begin{array}{llll}
1 & 1 & 0 & 1 \\
1 & 1 & 0 & 1 \\
0 & 0 & 0 & 0 \\
1 & 1 & 0 & 1
\end{array}\right)
$$

which can be factored as $\left(\begin{array}{llll}1 & 1 & 0 & 1\end{array}\right)^{T}\left(\begin{array}{llll}1 & 1 & 0 & 1\end{array}\right)$. By multiplying $\left(\begin{array}{llll}1 & 1 & 0 & 1\end{array}\right)$ with the vector of monomials $\left(\begin{array}{lllll}1 & \xi_{1} & \xi_{2} & \xi_{1} \xi_{2}\end{array}\right)^{T}$, this yields a spectral factor

$$
F\left(\xi_{1}, \xi_{2}\right)=1+\xi_{1}+\xi_{1} \xi_{2}
$$

In the first part of this section we have dealt with factorization of polynomial matrices, where the spectral factors were again required to be polynomial matrices. In general, for a given para-hermitian polynomial matrix, a necessary condition for the existence of a polynomial spectral factor is that $Z(i \omega) \geq 0$ for all $\omega \in \mathbb{R}^{n}$. For the case of one indeterminate this is also a sufficient condition, and several algorithms exist for obtaining polynomial spectral factors, see $[2,4,7,14,15]$. For $n>1$ the condition $Z(i \omega) \geq 0$ for all $\omega \in \mathbb{R}^{n}$ is no longer sufficient for the existence of a polynomial spectral factor. This situation changes if we allow the spectral factor $F(\xi)$ to be a rational matrix, i.e., $F(\xi) \in \mathbb{R}^{r \times q}(\xi)$. In that case, the requirement $Z(i \omega) \geq 0$ for all $\omega \in \mathbb{R}^{n}$ is a necessary and sufficient condition.

Theorem 4.2 Let $Z(\xi)$ be a $q \times q$ para-hermitian n-variable polynomial matrix. Let $\Phi(\zeta, \eta) \in \mathbb{R}^{q \times q}[\zeta, \eta]$ be any symmetric $2 n$-variable polynomial matrix such that $\Phi(-\xi, \xi)=$ $Z(\xi)$. Then the following three statements are equivalent:

(i) There exists an integer $r$ and an n-variable rational matrix $F(\xi) \in \mathbb{R}^{r \times q}(\xi)$ such that

$$
Z(\xi)=F^{T}(-\xi) F(\xi) .
$$

(ii) There exists an $n$-tuple of symmetric $2 n$-variable polynomial matrices $\Psi(\zeta, \eta)=$ $\left(\Psi_{1}(\zeta, \eta), . ., \Psi_{n}(\zeta, \eta)\right)$ with $\Psi_{i} \in \mathbb{R}_{s}^{q \times q}[\zeta, \eta], i=1, \ldots, n$, and a polynomial $p \in \mathbb{R}[\xi]$ such that

$$
\operatorname{mat}\left(\Phi^{\prime}-\dot{\Psi}\right) \geq 0
$$

with $\Phi^{\prime}(\zeta, \eta):=\Phi(\zeta, \eta) p(\zeta) p(\eta) ;$

(iii) $Z(i \omega) \geq 0$ for all $\omega \in \mathbb{R}^{n}$.

Assume that any of these conditions hold. Then an $F$ such that (14) holds can be computed as follows: find $a \Psi(\zeta, \eta)=\left(\Psi_{1}(\zeta, \eta), . ., \Psi_{n}(\zeta, \eta)\right)$ and a polynomial $p(\xi)$, such that (15) holds, and factorize

$$
\operatorname{mat}\left(\Phi^{\prime}-\dot{\Psi}\right)=\tilde{D}^{T} \tilde{D}
$$

where $\tilde{D}$ is a constant matrix with full row rank, say $r$. Define $D(\xi)$ as the polynomial matrix with coefficient matrix $\tilde{D}$ (i.e., $\operatorname{mat}(D)=\tilde{D}$ ). Finally define $F(\xi):=p(\xi)^{-1} D(\xi)$. 
Proof : The equivalence of (i) and (iii) is proved in [12]. ((i) $\Rightarrow$ (ii)) Let the polynomial $p(\xi)$ be the least common multiple of the denominators in the entries of $F(\xi)$. Then obviously $F(\xi)=p(\xi)^{-1} D(\xi)$ for some polynomial matrix $D(\xi)$. Now define $\hat{\Phi}(\zeta, \eta):=\Phi(\zeta, \eta) p(\zeta) p(\eta)-D^{T}(\zeta) D(\eta)$. Since $\hat{\Phi}(-\xi, \xi)=0$ there exists $\Psi(\zeta, \eta)=$ $\left(\Psi_{1}(\zeta, \eta), . ., \Psi_{n}(\zeta, \eta)\right)$ with $\Psi_{i} \in \mathbb{R}_{s}^{q \times q}[\zeta, \eta], i=1, \ldots, n$ such that $\hat{\Phi}(\zeta, \eta)=\dot{\Psi}(\zeta, \eta)$. This implies that

$$
\operatorname{mat}\left((\Phi(\zeta, \eta) p(\zeta) p(\eta)-\dot{\Psi}(\zeta, \eta))=\operatorname{mat}\left(D^{T}(\zeta) D(\eta)\right)\right.
$$

and since $\operatorname{mat}\left(D^{T}(\zeta) D(\eta)\right) \geq 0$ one obtains (ii).

$(($ ii $) \Rightarrow($ i $))$ : Factorize $\operatorname{mat}\left(\Phi^{\prime}-\dot{\Psi}\right)=\tilde{D}^{T} \tilde{D}$. Define a polynomial matrix $D(\xi)$ with coefficient matrix $\tilde{D}$ (i.e., $\operatorname{mat}(D)=\tilde{D})$. Then $\Phi^{\prime}(\zeta, \eta)-\dot{\Psi}(\zeta, \eta)=D(\zeta)^{T} D(\eta)$ and setting $\eta=-\zeta=\xi$ we get $Z(\xi)=\Phi(-\xi, \xi)=\left(p^{-1}(-\xi) D(-\xi)\right)^{T} p^{-1}(\xi) D(\xi)$ which implies (i) .

Remark 4.2 Condition (21) can be restated as $p(\zeta) \Phi(\zeta, \eta) p(\eta)-\dot{\Psi}(\zeta, \eta)=D^{T}(\zeta) D(\eta)$, equivalently, $\operatorname{div}\left(Q_{\Psi}(\ell)\right)-Q_{\Phi}\left(p\left(\frac{d}{d x}\right) \ell\right)=\left\|D\left(\frac{d}{d x}\right) \ell\right\|^{2}$ for all $\ell \in \mathfrak{C}^{\infty}\left(\mathbb{R}^{n}, \mathbb{R}^{q}\right)$. This says that the $n \mathrm{D}$ system behavior $\mathfrak{B}=\mathfrak{C}^{\infty}\left(\mathbb{R}^{n}, \mathbb{R}^{q}\right)$, written in image representation $w=\operatorname{Ip}\left(\frac{d}{d x}\right) \ell$, is $Q_{\Phi}$ dissipative, and has storage function $Q_{\Psi}(\ell)$ with corresponding dissipation rate $\left\|D\left(\frac{d}{d x}\right) \ell\right\|^{2}$.

In the inequality (15) the unknowns are the entries of $\operatorname{mat}\left(\Psi_{i}\right), i=1,2, \ldots n$, and the coefficients of $p$. Of course, the coefficients of $p$ appear quadratically in the inequality, and therefore (15) is no longer a linear matrix inequality. An additional complication is that the highest monomial degree appearing in $p(\xi)$ is unknown, so the number of unknowns in the inequality is also an unknown.

In the remainder of this section we will propose two different ideas for possible algorithms to solve the inequality (15).

Our first algorithm reduces the nonlinear problem to a linear eigenvalue problem. In order to avoid too cumbersome notation, we consider for this first algorithm only the case $n=2$. For the sake of simplicity, we will also assume that $\Phi(\zeta, \eta)$ can be chosen such that its coefficient matrix mat $(\Phi)$ is nonsingular. Let $p\left(\xi_{1}, \xi_{2}\right)$ be a real polynomial with highest monomial degree $\left(M_{1}, M_{2}\right)$ given by

$$
\begin{aligned}
& p\left(\xi_{1}, \xi_{2}\right)=p_{0,0}+p_{1,0} \xi_{1}+p_{2,0} \xi_{1}^{2}+\ldots+p_{M_{1}, 0} \xi_{1}^{M_{1}}+ \\
& p_{0,1} \xi_{2}+p_{1,1} \xi_{1} \xi_{2}+\ldots+p_{M_{1}, 1} \xi_{1}^{M_{1}} \xi_{2}+ \\
& \ldots+p_{0, M_{2}} \xi_{2}^{M_{2}}+p_{1, M_{2}} \xi_{1} \xi_{2}^{M_{2}}+\ldots+p_{M_{1}, M_{2}} \xi_{1}^{M_{1}} \xi_{2}^{M_{2}} \\
& =\left(\begin{array}{llll}
P_{0}^{T} & P_{1}^{T} & \ldots & P_{M_{2}}^{T}
\end{array}\right)\left(\begin{array}{llll}
U\left(\xi_{1}\right) & \xi_{2} U\left(\xi_{1}\right) & \ldots & \xi_{2}^{M_{2}} U\left(\xi_{1}\right)
\end{array}\right)^{T}
\end{aligned}
$$

where $P_{i}^{T}:=\left(p_{0, i}, p_{1, i} \ldots, p_{M_{1}, i}\right)$ for $i=0, \ldots, M_{2}$ and $U\left(\xi_{1}\right):=\left(1, \xi_{1}, \ldots, \xi_{1}^{M_{1}}\right)$. Let $I$ denote the $q \times q$ identity matrix, and for $i=0,1, \ldots N_{1}$, define shift operators $\bar{\sigma}_{i}$ acting on $P \in \mathbb{R}^{\left(M_{1}+1\right) \times 1}, P=\left(p_{0}, p_{1} \ldots, p_{M_{1}}\right)$ by

$$
\bar{\sigma}_{i}(P)=\left(\begin{array}{ccccccccccc}
0_{q \times q i} & \vdots & p_{0} I & \vdots & p_{1} I & \vdots & \ldots & \vdots & p_{M_{1}} I & \vdots & 0_{q \times\left(N_{1}-i\right) q}
\end{array}\right)^{T},
$$


and a $\left(M_{1}+N_{1}+1\right)\left(M_{2}+1\right) q \times\left(N_{1}+1\right) q$ matrix $\Sigma$ by

$$
\Sigma:=\left(\begin{array}{cccc}
\bar{\sigma}_{0}\left(P_{0}\right) & \bar{\sigma}_{1}\left(P_{0}\right) & \ldots & \bar{\sigma}_{N_{1}}\left(P_{0}\right) \\
\bar{\sigma}_{0}\left(P_{1}\right) & \bar{\sigma}_{1}\left(P_{1}\right) & \cdots & \bar{\sigma}_{N_{1}}\left(P_{1}\right) \\
\vdots & \vdots & \vdots & \vdots \\
\bar{\sigma}_{0}\left(P_{M_{2}}\right) & \bar{\sigma}_{1}\left(P_{M_{2}}\right) & \ldots & \bar{\sigma}_{N_{1}}\left(P_{M_{2}}\right)
\end{array}\right)
$$

For $i=0,1, \ldots, N_{2}$ define

$$
\hat{\sigma}_{i}(\Sigma):=\left(\begin{array}{c}
0_{\left(M_{1}+N_{1}+1\right) q i \times\left(N_{1}+1\right) q} \\
\Sigma \\
0_{\left(M_{1}+N_{1}+1\right)\left(N_{2}-i\right) q \times\left(N_{1}+1\right) q}
\end{array}\right) .
$$

Finally, define a $\left(M_{1}+N_{1}+1\right)\left(M_{2}+N_{2}+1\right) q \times\left(N_{1}+1\right)\left(N_{2}+1\right) q$ matrix $M$ by

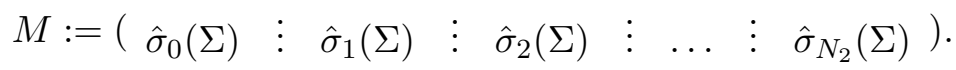

It is a matter of straightforward calculation to check that the coefficient matrix of $p(\zeta) \Phi(\zeta, \eta) p(\eta)$ is $M \operatorname{mat}(\Phi) M^{T}$. Define

$$
A=\left(\begin{array}{cc}
-\operatorname{mat}(\dot{\Psi}) & M \\
M^{T} & -\operatorname{mat}(\Phi)^{-1}
\end{array}\right) \text { and } B=\left(\begin{array}{cc}
M \operatorname{mat}(\Phi) M^{T}-\operatorname{mat}(\dot{\Psi}) & 0 \\
0 & -\operatorname{mat}(\Phi)^{-1}
\end{array}\right) .
$$

Then we have

$$
\left(\begin{array}{cc}
I & M \operatorname{mat}(\Phi) \\
0 & I
\end{array}\right) A\left(\begin{array}{cc}
I & 0 \\
\operatorname{mat}(\Phi) M^{T} & I
\end{array}\right)=B
$$

Note that $M \operatorname{mat}(\Phi) M^{T}-\operatorname{mat}(\dot{\Psi})$ is the Schur-complement of $-\operatorname{mat}(\Phi)^{-1}$ in $B$ and that the signatures of $A$ and $B$ are equal. Thus we find that the (nonlinear) matrix inequality $M \operatorname{mat}(\Phi) M^{T}-\operatorname{mat}(\dot{\Psi}) \geq 0$ has a solution if and only if $A$ has the same number of negative eigenvalues as the matrix $-\operatorname{mat}(\Phi)^{-1}$. It is important to note that $A$ depends linearly on the unknowns. The solution of this linear eigenvalue problem will provide a $p$ and a $\Psi(\zeta, \eta)$ such that $\operatorname{mat}\left(\Phi^{\prime}-\operatorname{mat}(\dot{\Psi})\right)$ is positive semidefinite. Moreover, we can then construct the spectral factor $F(\xi)$ as explained in theorem 4.2.

We conclude this section with our second conceptual algorithm to solve the nonlinear inequality (15). In the following, we will denote the unknowns appearing in (15) by $\alpha_{1}, \alpha_{2}, \ldots, \alpha_{k}$ (where $k$ is also unknown), and collect them in a single vector $\alpha \in \mathbb{R}^{k}$. The inequality (15) can be written as $S\left(\alpha_{1}, \ldots, \alpha_{k}\right) \geq 0$, where $S(\alpha)$ is a real symmetric matrix function, say of size $N$. In the following, we will reduce this inequality to checking feasability of a semi-algebraic set. In order to proceed, let $L_{\alpha}(\lambda):=\operatorname{det}(S(\alpha)-\lambda I)$ be the characteristic polynomial of $S(\alpha)$. Write

$$
L_{\alpha}(\lambda)=\lambda^{N}+s_{N-1}(\alpha) \lambda^{N-1}+\cdots+s_{1}(\alpha) \lambda+s_{0}(\alpha) .
$$

The coefficients $s_{i}(\alpha)$ are all real polynomials in the indeterminate $\alpha$. Since $S(\alpha)$ is symmetric for all $\alpha$, the roots of $L_{\alpha}$ are all real. Our aim is to find $\alpha \in \mathbb{R}^{k}$ such that all 
these roots are nonnegative. In order to find such $\alpha$ 's, consider the polynomial $L_{\alpha}(-\lambda)$. Obviously, the number of negative roots of $L_{\alpha}(\lambda)$ is equal to the number of positive roots of $L_{\alpha}(-\lambda)$. By Descartes' rule of signs, the number of positive eigenvalues of $L_{\alpha}(-\lambda)$ is equal to the number of sign changes between the consecutive nonzero coefficients in this polynomial. We therefore conclude that $L_{\alpha}(\lambda)$ has only nonnegative roots if and only if $(-1)^{N+i} s_{i}(\alpha) \geq 0$ for all $i=1, \ldots, N-1$. Hence, the inequality (15) has a solution $\alpha$ if and only if the semi-algebraic set

$$
U:=\left\{\alpha \in \mathbb{R}^{k} \mid(-1)^{N+i} s_{i}(\alpha) \geq 0, i=1, \ldots, N-1\right\} .
$$

is not empty. A feasible point $\alpha$ in $U$ correspond to a polynomial $p$ and to a $\Psi(\zeta, \eta)$ such that $\operatorname{mat}\left(\Phi^{\prime}-\dot{\Psi}\right)$ is positive semidefinite. Moreover after finding a feasable $\alpha$, we can construct a spectral factor $F(\xi)$ as explained in theorem 4.2. Unfortunately, even if we know that the semialgebraic set $U$ is not empty, in general, to find a feasible point is a difficult problem. However recent developments have led to an approach using semidefinite programming to deal with semialgebraic sets $[9,8]$.

Since the degree of $p$ is unknown, we may have to execute these algorithms for different degrees of $p$.

Another important difference between the case $n=1$ and $n>1$ is that, under the condition that a spectral factor exists, in the multidimensional case it is not always possible to find a square spectral factor, not even in the scalar case with $n=2$, see $[6,1]$. In the case that $n=1$ this is always possible.

\section{Sum of squares}

Let $Z(\xi) \in \mathbb{R}[\xi]$ be a polynomial in $n$ indeterminates, and assume that $Z(\xi)=F^{T}(-\xi) F(\xi)$ for some $n$-variable polynomial row vector $F(\xi)$. Substituting $\xi=i \omega$ yields $Z(i \omega)=$ $F^{T}(-i \omega) F(i \omega)$. If $F(i \omega)$ is decomposed into real and imaginary parts as $F(i \omega)=$ $A(\omega)+i B(\omega)$, we have $Z(i \omega)=A^{2}(\omega)+B^{2}(\omega)$. Thus, one can see that representation as sum of squares is at the heart of the problem of spectral factorization, and therefore we expect that similar techniques can be used in order to treat the sum of squares problem.

A given $n$-variable polynomial $q \times q$ matrix $Z(\xi)$ is called positive semi-definite if $Z(\xi)^{T}=Z(\xi)$ and $Z(\xi) \geq 0$ for all $\xi \in \mathbb{R}^{n}$. In the following, we will associate with $Z(\xi)$ symmetric, $2 n$-variable polynomial $q \times q$ matrices $\Phi(\zeta, \eta)$ with the property that

$$
\Phi(\xi, \xi)=Z(\xi) .
$$

For a given $Z$ there are always infinitely many $\Phi$ 's such that this property holds. One possible choice of $\Phi$ such that (17) holds is given by

$$
\Phi(\zeta, \eta):=\frac{1}{2}\left(Z^{T}(\zeta)+Z(\eta)\right)
$$

Since $\Phi(\eta, \zeta)^{T}=\frac{1}{2}\left(Z(\eta)+Z^{T}(\zeta)\right)=\Phi(\zeta, \eta), \Phi(\zeta, \eta)$ is symmetric. Using $Z^{T}(\xi)=Z(\xi)$, this $\Phi$ indeed satisfies (17).

For a given $n$-variable polynomial matrix $Z(\xi)$, it is possible to characterize all symmetric $2 n$-variable polynomial matrices $\Phi(\zeta, \eta)$ such that (17) holds. The procedure to do that is analogous to the one described in section 3 to characterize all symmetric $2 n$ variable polynomial matrices $\Phi(\zeta, \eta)$ such that $\Phi(-\xi, \xi)=Z(\xi)$. The only difference is 
that, in the present case, there is no alternating sign pattern: the signs of the blocks on the various anti-diagonals are all positive. We omit the details here.

In the following we will use skew-symmetric $2 n$-variable polynomial matrices. A given $\Phi \in \mathbb{R}^{q \times q}[\zeta, \eta]$ will be called skew-symmetric if $\Phi(\zeta, \eta)^{T}=-\Phi(\eta, \zeta)$. It is easily verified that $\Phi$ is skew-symmetric if and only if is coefficient matrix is skew-symmetric, i.e., $\operatorname{mat}(\Phi)^{T}=-\operatorname{mat}(\Phi)$.

Lemma 5.1 Let $\Phi \in \mathbb{R}_{s}^{q \times q}[\zeta, \eta]$. Then the following statements are equivalent:

(i) $\Phi(\xi, \xi)=0$,

(ii) there exist an $n$-tuple $\Psi(\zeta, \eta)=\left(\left(\Psi_{1}(\zeta, \eta), . ., \Psi_{n}(\zeta, \eta)\right)\right.$ with $\Psi_{i} \in \mathbb{R}^{q \times q}[\zeta, \eta], i=$ $1, \ldots, n$, skew-symmetric such that

$$
\Phi(\zeta, \eta)=\left(\zeta_{1}-\eta_{1}\right) \Psi_{1}(\zeta, \eta)+\ldots+\left(\zeta_{n}-\eta_{n}\right) \Psi_{n}(\zeta, \eta) .
$$

Proof : $(i) \Rightarrow(i i)$ Define $\Phi^{\prime}(\zeta, \eta):=\Phi(\zeta,-\eta)$. Clearly $\Phi^{\prime}(-\xi, \xi)=0$, so there exists an $n$-tuple $\Psi(\zeta, \eta)=\left(\Psi_{1}(\zeta, \eta), \ldots, \Psi_{n}(\zeta, \eta)\right)$ such that $\Phi^{\prime}(\zeta, \eta)=\left(\zeta_{1}+\eta_{1}\right) \Psi_{1}(\zeta, \eta)+\ldots+$ $\left(\zeta_{n}+\eta_{n}\right) \Psi_{n}(\zeta, \eta)$. This implies (18). We see that the $\Psi_{i}$ can be chosen skew-symmetric. Indeed, using that $\Phi(\zeta, \eta)^{T}=\Phi(\eta, \zeta)$ it can be shown that $\Phi(\zeta, \eta)=\left(\zeta_{1}-\eta_{1}\right) \Psi_{1}^{\prime}(\zeta, \eta)+$ $\ldots+\left(\zeta_{n}-\eta_{n}\right) \Psi_{n}^{\prime}(\zeta, \eta)$, with $\Psi_{i}^{\prime}(\zeta, \eta):=\frac{1}{2}\left(\Psi_{i}(\zeta, \eta)-\Psi_{i}(\eta, \zeta)^{T}\right)$ skew-symmetric.

$($ ii $) \Rightarrow($ i) Obvious by substitution.

Analogous to theorem 4.1, we obtain the following theorem for SOS.

Theorem 5.1 Let $Z(\xi) \in \mathbb{R}^{q \times q}[\xi]$ be a positive semidefinite $n$-variable polynomial matrix. Let $\Phi(\zeta, \eta) \in \mathbb{R}_{s}^{q \times q}[\zeta, \eta]$ be such that $\Phi(\xi, \xi)=Z(\xi)$. Then the following statements are equivalent:

(i) $Z(\xi)$ is sum of squares, i.e. there exists a positive integer $r$ and an $r \times q$ polynomial matrix $F(\xi)$ such that

$$
Z(\xi)=F^{T}(\xi) F(\xi) .
$$

(ii) There exist an $n$-tuple of skew-symmetric $2 n$-variable polynomial matrices $\Psi(\zeta, \eta)=$ $\left(\Psi_{1}(\zeta, \eta), . ., \Psi_{n}(\zeta, \eta)\right)$ with $\Psi_{i} \in \mathbb{R}^{q \times q}[\zeta, \eta], i=1, \ldots, n$ such that

$$
\operatorname{mat}(\Phi-\bar{\Psi}) \geq 0
$$

$$
\text { with } \bar{\Psi}(\zeta, \eta):=\left(\zeta_{1}-\eta_{1}\right) \Psi_{1}(\zeta, \eta)+\left(\zeta_{2}-\eta_{2}\right) \Psi_{2}(\zeta, \eta)+\ldots+\left(\zeta_{n}-\eta_{n}\right) \Psi_{n}(\zeta, \eta) .
$$

(iii) There exists a symmetric $2 n$-variable polynomial matrix $\hat{\Phi}(\zeta, \eta)$ such that $\hat{\Phi}(\xi, \xi)=$ $Z(\xi)$ and $\operatorname{mat}(\hat{\Phi}) \geq 0$.

Assume that any of these conditions hold. Then an F such that (19) holds can be computed as follows: find $\Psi(\zeta, \eta)=\left(\Psi_{1}(\zeta, \eta), . ., \Psi_{n}(\zeta, \eta)\right)$, such that (20) holds, and factorize

$$
\operatorname{mat}(\Phi-\bar{\Psi})=\tilde{F}^{T} \tilde{F},
$$

where $\tilde{F}$ is a constant matrix with row rank, say $r$. Then the polynomial matrix $F(\xi)$ with coefficient matrix $\tilde{F}$ (i.e., $\operatorname{mat}(F)=\tilde{F}$ ) satisfies (19). 
Proof : $(i) \Rightarrow(i i)$ Clearly $\Phi(\xi, \xi)-F^{T}(\xi) F(\xi)=0$. By proposition 5.1, there exists a vector of skew-symmetric $2 n$-variable polynomial matrices $\Psi(\zeta, \eta)=\left(\Psi_{1}(\zeta, \eta), . ., \Psi_{n}(\zeta, \eta)\right)$ with $\Psi_{i} \in \mathbb{R}^{q \times q}[\zeta, \eta]$ such that $\Phi(\zeta, \eta)-F^{T}(\zeta) F(\eta)=\bar{\Psi}(\zeta, \eta)=\left(\zeta_{1}-\eta_{1}\right) \Psi_{1}(\zeta, \eta)+\ldots+$ $\left(\zeta_{n}-\eta_{n}\right) \Psi_{n}(\zeta, \eta)$. This equality can be written in terms of the associated coefficient matrices as $\operatorname{mat}(\Phi)-\tilde{F}^{T} \tilde{F}=\operatorname{mat}(\bar{\Psi})$, where $\tilde{F}$ is the coefficient matrix of $F(\xi)$. This implies (20) since $\tilde{F}^{T} \tilde{F} \geq 0$.

$($ ii $) \Rightarrow(i)$ : Since $\operatorname{mat}(\Phi-\bar{\Psi})$ is a symmetric positive semidefinite matrix we can factorize it as $\operatorname{mat}(\Phi-\bar{\Psi})=\tilde{F}^{T} \tilde{F}$, with row rank, say $r$. Now let $F(\xi)$ be the $n$ variable polynomial matrix with coefficient matrix $\tilde{F}$. Then we obtain $\Phi(\zeta, \eta)-\bar{\Psi}(\zeta, \eta)=$ $F^{T}(\zeta) F(\eta)$. By taking $\zeta=\eta=\xi$ we then obtain $Z(\xi)=\Phi(\xi, \xi)=F^{T}(\xi) F(\xi)$.

$(i) \Rightarrow($ iii $)$ Define $\hat{\Phi}(\zeta, \eta):=F^{T}(\zeta) F(\eta)$.

(iii) $\Rightarrow($ i $)$ Factor mat $(\hat{\Phi})=\tilde{F}^{T} \tilde{F}$ and define $F(\xi)$ as the $n$-variable polynomial matrix such that $\operatorname{mat}(F)=\tilde{F}$.

We note that, in a different form, condition (iii) in the above theorem can also be found in [3] and [13] in the context of sum of squares of real polynomials.

As in the previous section, in order to be able to apply the above theorem, we need to be able to express $(20)$ as an LMI. Thus we need to express mat $(\bar{\Psi})$ in terms of shifts of the coefficient matrices mat $\left(\Psi_{i}\right)$. It is easy to see that $\operatorname{mat}\left(\left(\zeta_{i}-\eta_{i}\right) \Psi_{i}(\zeta, \eta)\right)=$ $\left(\sigma_{i, D}-\sigma_{i, R}\right)\left(\operatorname{mat}\left(\Psi_{i}\right)\right)$ for $i=1, \ldots, n$ and therefore the inequality $(20)$ can be expressed as

$\operatorname{mat}(\Phi)-\left(\sigma_{1, D}-\sigma_{1, R}\right)\left(\operatorname{mat}\left(\Psi_{1}\right)\right)-\left(\sigma_{2, D}-\sigma_{2, R}\right)\left(\operatorname{mat}\left(\Psi_{2}\right)\right)-\cdots-\left(\sigma_{n, D}-\sigma_{n, R}\right)\left(\operatorname{mat}\left(\Psi_{n}\right)\right) \geq 0$.

If the highest monomial degree of $\Phi(\zeta, \eta)$ is equal to $\left(N_{1}, N_{2}, \ldots, N_{n}\right)$ then, as before, $\operatorname{mat}\left(\Psi_{i}\right)$ has size $n_{i}:=q N_{i} \prod_{j=1, j \neq i}^{n}\left(N_{j}+1\right)(i=1,2 \ldots, n)$. Since the $\Psi_{i}$, or equivalently the matrices $\operatorname{mat}\left(\Psi_{i}\right)$, can always be taken to be skew-symmetric, the actual number of unknowns in the LMI (20) is equal to $\sum_{i=1}^{n} \frac{n_{i}\left(n_{i}-1\right)}{2}$.

We illustrate the use of the previous theorem for SOS by means of the following example:

Example 5.1 Let $Z(\xi)=\left(\begin{array}{cc}1-2 \xi_{1}+\xi_{1}^{2}+\left(\xi_{1}^{2} \xi_{2}\right)^{2} & \xi_{1}-\xi_{1}^{2} \\ \xi_{1}-\xi_{1}^{2} & 1-2 \xi_{1}+2 \xi_{1}^{2}+\left(\xi_{1}^{2} \xi_{2}\right)^{2}\end{array}\right)$. We want to find, if these exist, a positive integer $r$ and an $F(\xi) \in \mathbb{R}^{r \times 2}[\xi]$ such that $Z(\xi)=$ $F^{T}(\xi) F(\xi)$.

We first construct $\Phi(\zeta, \eta) \in \mathbb{R}_{s}^{2 \times 2}[\zeta, \eta]$ such that $\Phi(\xi, \xi)=Z(\xi)$. This condition can be expressed in terms of the appropriate anti-diagonals of the coefficient matrix of $\Phi$, with positive signs only. Thus we find that we can take $\Phi(\zeta, \eta)$ equal to

$\left(I, I \zeta_{1}, I \zeta_{1}^{2} \mid I \zeta_{2}, I \zeta_{1} \zeta_{2}, I \zeta_{1}^{2} \zeta_{2}\right)\left(\begin{array}{cccccc|cccccc}1 & 0 & -1 & 1 & \frac{1}{2} & -1 & 0 & 0 & 0 & 0 & 0 & 0 \\ 0 & 1 & 0 & -1 & 0 & 1 & 0 & 0 & 0 & 0 & 0 & 0 \\ -1 & 0 & 0 & 0 & 0 & 0 & 0 & 0 & 0 & 0 & 0 & 0 \\ 1 & -1 & 0 & 0 & 0 & 0 & 0 & 0 & 0 & 0 & 0 & 0 \\ \frac{1}{2} & 0 & 0 & 0 & 0 & 0 & 0 & 0 & 0 & 0 & 0 & 0 \\ -1 & 1 & 0 & 0 & 0 & 0 & 0 & 0 & 0 & 0 & 0 & 0 \\ \hline 0 & 0 & 0 & 0 & 0 & 0 & 0 & 0 & 0 & 0 & 0 & 0 \\ 0 & 0 & 0 & 0 & 0 & 0 & 0 & 0 & 0 & 0 & 0 & 0 \\ 0 & 0 & 0 & 0 & 0 & 0 & 0 & 0 & 0 & 0 & 0 & 0 \\ 0 & 0 & 0 & 0 & 0 & 0 & 0 & 0 & 0 & 0 & 0 & 0 \\ 0 & 0 & 0 & 0 & 0 & 0 & 0 & 0 & 0 & 0 & 1 & 0 \\ 0 & 0 & 0 & 0 & 0 & 0 & 0 & 0 & 0 & 0 & 0 & 1\end{array}\right)\left(\begin{array}{c}I \\ I \eta_{1} \\ I \eta_{1}^{2} \\ -- \\ I \eta_{2} \\ I \eta_{1} \eta_{2} \\ I \eta_{1}^{2} I \eta_{2}\end{array}\right)$, 
where $I$ denotes the $2 \times 2$ identity matrix. Next, we search for $\Psi_{1}(\zeta, \eta), \Psi_{2}(\zeta, \eta) \in$ $\mathbb{R}^{2 \times 2}[\zeta, \eta]$, skew-symmetric, with highest monomial degrees $(2,0)$ and $(1,1)$ respectively.

Their (unknown) coefficient matrices are given by

$$
\begin{aligned}
\operatorname{mat}\left(\Psi_{1}\right) & =\left(\begin{array}{cccc|cccc}
0 & a_{1} & a_{2} & a_{3} & a_{4} & a_{5} & a_{6} & a_{7} \\
-a_{1} & 0 & a_{8} & a_{9} & a_{10} & a_{11} & a_{12} & a_{13} \\
-a_{2} & -a_{8} & 0 & a_{14} & a_{15} & a_{16} & a_{17} & a_{18} \\
-a_{3} & -a_{9} & -a_{14} & 0 & a_{19} & a_{20} & a_{21} & a_{22} \\
\hline-a_{4} & -a_{10} & -a_{15} & -a_{19} & 0 & a_{23} & a_{24} & a_{25} \\
-a_{5} & -a_{11} & -a_{16} & -a_{20} & -a_{23} & 0 & a_{26} & a_{27} \\
-a_{6} & -a_{12} & -a_{17} & -a_{21} & -a_{24} & -a_{26} & 0 & a_{29} \\
-a_{6} & -a_{13} & -a_{18} & -a_{22} & -a_{25} & -a_{27} & -a_{29} & 0
\end{array}\right), \\
\operatorname{mat}\left(\Psi_{2}\right) & =\left(\begin{array}{cccccc}
0 & b_{1} & b_{2} & b_{3} & b_{4} & b_{5} \\
-b_{1} & 0 & b_{6} & b_{7} & b_{8} & b_{9} \\
-b_{2} & -b_{6} & 0 & b_{10} & b_{11} & b_{12} \\
-b_{3} & -b_{7} & -b_{10} & 0 & b_{13} & b_{14} \\
-b_{4} & -b_{8} & -b_{11} & -b_{13} & 0 & b_{15} \\
-b_{5} & -b_{9} & -b_{12} & -b_{14} & -b_{15} & 0
\end{array}\right)
\end{aligned}
$$

and the LMI we want to solve is

$$
\operatorname{mat}(\Phi)-\left(\sigma_{1, D}-\sigma_{1, R}\right)\left(\operatorname{mat}\left(\Psi_{1}\right)\right)-\left(\sigma_{2, D}-\sigma_{2, R}\right)\left(\operatorname{mat}\left(\Psi_{2}\right)\right) \geq 0 .
$$

One solution of this inequality is given by

$$
\operatorname{mat}\left(\Psi_{1}\right)=\left(\begin{array}{cccc|cccc}
0 & 0 & -\frac{1}{2} & 1 & 0 & 0 & 0 & 0 \\
0 & 0 & 0 & -1 & 0 & 0 & 0 & 0 \\
\frac{1}{2} & 0 & 0 & 0 & 0 & 0 & 0 & 0 \\
-1 & 1 & 0 & 0 & 0 & 0 & 0 & 0 \\
\hline 0 & 0 & 0 & 0 & 0 & 0 & 0 & 0 \\
0 & 0 & 0 & 0 & 0 & 0 & 0 & 0 \\
0 & 0 & 0 & 0 & 0 & 0 & 0 & 0 \\
0 & 0 & 0 & 0 & 0 & 0 & 0 & 0
\end{array}\right),
$$

and $\operatorname{mat}\left(\Psi_{2}\right)$ the zero matrix. This yields

$$
\operatorname{mat}(\Phi-\bar{\Psi})=\left(\begin{array}{cccccc|cccccc}
1 & 0 & -1 & 1 & 0 & 0 & 0 & 0 & 0 & 0 & 0 & 0 \\
0 & 1 & 0 & -1 & 0 & 0 & 0 & 0 & 0 & 0 & 0 & 0 \\
-1 & 0 & 1 & -1 & 0 & 0 & 0 & 0 & 0 & 0 & 0 & 0 \\
1 & -1 & -1 & 2 & 0 & 0 & 0 & 0 & 0 & 0 & 0 & 0 \\
0 & 0 & 0 & 0 & 0 & 0 & 0 & 0 & 0 & 0 & 0 & 0 \\
0 & 0 & 0 & 0 & 0 & 0 & 0 & 0 & 0 & 0 & 0 & 0 \\
\hline 0 & 0 & 0 & 0 & 0 & 0 & 0 & 0 & 0 & 0 & 0 & 0 \\
0 & 0 & 0 & 0 & 0 & 0 & 0 & 0 & 0 & 0 & 0 & 0 \\
0 & 0 & 0 & 0 & 0 & 0 & 0 & 0 & 0 & 0 & 0 & 0 \\
0 & 0 & 0 & 0 & 0 & 0 & 0 & 0 & 0 & 0 & 0 & 0 \\
0 & 0 & 0 & 0 & 0 & 0 & 0 & 0 & 0 & 0 & 1 & 0 \\
0 & 0 & 0 & 0 & 0 & 0 & 0 & 0 & 0 & 0 & 0 & 1
\end{array}\right) \geqslant 0 .
$$

The rank of this matrix is equal to 4 , and it can be factorized as $\operatorname{mat}(\Phi-\bar{\Psi})=\tilde{F}^{T} \tilde{F}$, 
with $\tilde{F}$ given below. This gives

$$
F(\xi)=\left(\begin{array}{cc|cc|cc|cc|cc|cc}
1 & 0 & -1 & 1 & 0 & 0 & 0 & 0 & 0 & 0 & 0 & 0 \\
0 & 1 & 0 & -1 & 0 & 0 & 0 & 0 & 0 & 0 & 0 & 0 \\
\hline 0 & 0 & 0 & 0 & 0 & 0 & 0 & 0 & 0 & 0 & 1 & 0 \\
0 & 0 & 0 & 0 & 0 & 0 & 0 & 0 & 0 & 0 & 0 & 1
\end{array}\right)\left(\begin{array}{c}
I \\
I \xi_{1} \\
I \xi_{1}^{2} \\
-- \\
I \xi_{2} \\
I \xi_{1} \xi_{2} \\
I \xi_{1}^{2} I \xi_{2}
\end{array}\right),
$$

Hence,

$$
Z(\xi)=F^{T}(\xi) F(\xi)=\left(\begin{array}{cc|cc}
1-\xi_{1} & 0 & \xi_{1}^{2} \xi_{2} & 0 \\
\xi_{1} & 1-\xi_{1} & 0 & \xi_{1}^{2} \xi_{2}
\end{array}\right)\left(\begin{array}{cc|cc}
1-\xi_{1} & 0 & \xi_{1}^{2} \xi_{2} & 0 \\
\xi_{1} & 1-\xi_{1} & 0 & \xi_{1}^{2} \xi_{2}
\end{array}\right)^{T}
$$

\section{Conclusions}

In this paper we have presented a new framework in which is possible to develop algorithms to address the problems of spectral factorization and sum of squares, even for the matrix case, with any number of indeterminates. The algorithms are based on the idea of associating with the ( $n$-variable) polynomial matrix $Z$ to be factored, a $2 n$-variable polynomial matrix $\Phi$. For the case $n=2$ explicit formulas for such $\Phi$ have been given. This allows us to relate the problem of spectral factorization with the theory of multidimensional dissipative systems, and give, in most cases, a physical interpretation of the elements of the algorithms.

We expect that this approach will provide further results in this area. Future research will treat, for instance, the presense of uniform denominators in the spectral factorization problem (see also [16]), the reduction of the number of unknowns in the matrix inequalities presented, rational factors for SOS, and so on.

\section{References}

[1] S. Basur, 'On spectral factorization in multidimensions'. The Second International Workshop on Multidimensional (nD) Systems (Czocha Castle, 2000), 39-46, Tech. Univ. Press, Zielona Gra, 2000. 93B17 (15A23)

[2] F.M. Callier, "On polynomial spectral factorization by symmetric factor extraction", IEEE Trans. Aut. Contr., vol. 30, pp. 453-464, 1985.

[3] M.D. Choi, T. Y. Lam and B. Reznick, "Sums of squares of real polynomials", Proc. Sympos. Pure Math., Vol. 58, American Mathematical Society (1995), p. 103-126.

[4] W.A. Coppel, Linear Systems, Notes in Pure Mathematics, Australian National University, vol. 6, 1972.

[5] J. Ježek and V. Kučera, "Efficient Algorithm for Matrix Spectral Factorization", Automatica, vol. 21, no. 6, pp. 663-669, 1985.

[6] A. Kummert, , "Spectral factorization of two-variable para-hermitian polynomial matrices", Multidimensional Systems and Signal processing., vol. 1, pp. 327-339, 1990. 
[7] H. Kwakernaak, and M. Šebek, "Polynomial $J$-spectral factorization", IEEE Trans. Aut. Contr., vol. 39, no. 2, pp. 315-328,1994.

[8] P.A. Parrilo, B. Sturmfels, "Minimizing polynomial functions", In Algorithmic and quantitative real algebraic geometry, DIMACS Series in Discrete Mathematics and Theoretical Computer Science, Vol. 60, pp. 83-99, AMS, 2003.

[9] P.A. Parrilo, "Semidefinite programming relaxations for semialgebraic problems", Algebraic and geometric methods in discrete optimization, Vol.96, pp. 293-320, 2003.

[10] A. Papachristodoulou and S. Prajna, " On the construction of Lyapunov functions using the sum of squares decomposition", Proceedings of the 39th IEEE Conference on Desition and Control, pp. 3482 - 3487, December 2000.

[11] H.K. Pillai and S. Shankar, 'A behavioral approach to control of distributed systems', SIAM J. Control Optim., Vol. 37, No. 2, pp. 388 - 408, 1998.

[12] H. K. Pillai and J.C. Willems, "Lossless and disipative distributed systems", SIAM J. Contr. Optim., Vol. 40, No 5, pp. 1406 - 1430, 2002.

[13] V. Powers and T. Wörmann, "An algorithm for sums of squares of real polynomials", J. Pure Appl. Algebra, Journal of Pure and Applied Algebra,Vol.127, pp. 99-104, 1998.

[14] A.C.M. Ran and L. Rodman, "Factorization of matrix polynomials with symmetries", IMA Preprint Series, no. 993, 1992.

[15] P. Rapisarda and H.L. Trentelman, "New Algorithms for Polynomial J-Spectral Factorization", Math. Control Signals Systems 12:24-61, 1999.

[16] B. Reznick, "Uniform denominators in Hilbert's seventeenth problem ", Mathematische Zeitschrift, 220 (1995), no. 1, 75-97.

[17] N. Wiener, "Extrapolation, interpolation, and smoothing of stationary time series with engineering applications", The Technology Press of the Massachusetts Institute of Technology, Cambridge, Mass, pp. +163, 1949.

[18] J.C. Willems and H.L. Trentelman, "On quadratic differential forms", SIAM J. Contr. Optim., Vol. 36, No 5, pp. 1703 - 1749, 1998. 